\title{
COMETARY PHOTOMETRY
}

\author{
D. JEWITT \\ Institute for Astronomy \\ University of Hawaii \\ 2680 Woodlawn Drive \\ Honolulu HI 96822
}

\begin{abstract}
The study of comets using modern optical photometric techniques is reviewed. Particular emphasis is given to the physical constraints imposed on the nature of comets by photometric data. The photometric study of bare nuclei and of active comets is discussed.
\end{abstract}

\section{Introduction}

In this review I will primarily discuss photometry of comets at optical wavelengths. The review will concentrate on the application of linear array photometers to physical problems of interest in the study of comets, rather than on the detailed techniques or methodology of photometry. The practical problems encountered in cometary photometry span the range of problems found in both stellar (point-source) and galactic (surface) photometry, with the added complication that the target objects are in motion with respect to background stars. Historically, visual and photographic observers have produced a large fraction of published cometary photometry. These observational methods are specifically excluded from the present review, since the retina and the photographic emulsion are both non-linear detectors, and they introduce many difficult problems into cometary photometry. An excellent account of the photometric properties of the eye may be found in Padgham and Saunders (1975), while visual photometry of comets has been reviewed recently by Meisel and Morris (1982). Photographic photometry of comets is summarized by Roemer (1966a, 1966b, 1976). The highly profitable application of photomultipliers to cometary photometry has been discussed in detail by A'Hearn (1983). The present review excludes photometry of thermally emitted radiation (wavelengths $\lambda>$ $2-3 \mu \mathrm{m}$ ) and of radiation scattered at $\lambda<0.30 \mu \mathrm{m}$, since the IR and UV wavelengths are discussed in independent reviews. However, advances in detector technology, particularly the advent of infrared arrays, blur the differences between optical and IR photometry, and much of the present material is applicable at non-visual wavelengths. 
The worst impression for a review to give is that the reviewed field has been worked and reworked so thoroughly by previous generations of scientists, that the subject is basically "all used up" and, by implication, closed to further useful exploration. This is definitely not the case with cometary photometry, and in this review I will deliberately stress the unknowns in this field. The review is intended to be a self contained introduction to the field of cometary photometry, as well as an up-to-date summary of the useful literature.

\subsection{BACKGROUND}

By tradition, photometry of comets is usually reported in terms of the "total magnitude" (often denoted $m_{1}$ ) and the "nuclear magnitude" (often denoted $m_{2}$ ). Use of $m_{1}$ and $m_{2}$ was formally advocated by IAU Commission 20 in 1970 . Unfortunately, neither $m_{1}$ nor $m_{2}$ has a realizable physical definition, except in special cases, such as that of a stellar (point source) comet, for which $m_{l}=m_{2}$. Both "total magnitude" and "nuclear magnitude" are misnomers for quantities with imprecise practical definitions. The total magnitude, $m_{l}$, is poorly defined, because the coma is not a discrete, sharpedged structure, but instead fades gradually into the background light. Estimates of $m_{l}$ depend strongly on the ability of the photometer to separate the outer coma from the background (sky) brightness. The nuclear magnitude, $m_{2}$, is widely recognized as having little to do with the cometary nucleus (e.g., Sekanina 1976). When applied to extended comets, $m_{2}$ is supposed to denote the magnitude of the "central condensation", a perceived bright core or disk at the center of the coma. Little evidence exists to suggest that the central condensation is generally more than a psycho-physiological phenomenon, presumably caused by the steep surface brightness gradients found at the photo-centers of active comets (see $\$ 4.3$ and $\$ 4.4$ ). Except in the rarest of cases, the "total magnitude" and the "nuclear magnitude" cannot be accurately measured, and physical theories based on these quantities must be regarded with suspicion.

Sensible photometry of resolved comets must include explicit mention of the region of the coma that is measured, if the photometry is to be quantitatively useful. In this review, the magnitude of an extended comet is written $m_{\lambda}(p)$, where $p$ ["] is the radius of the circular photometry aperture and where $\lambda$ denotes the wavelength or filter employed. Magnitudes measured within an annulus are written $m_{\lambda}\left(p_{1}, p_{2}\right)$, where $p_{1}$ and $p_{2}$ are the inner and outer radii of the annulus, respectively. The advantage conveyed by this nomenclature is that ambiguities concerning the measured portion of the comet are eliminated. For example, $m_{R}\left(5^{\prime \prime}, 10^{\prime \prime}\right)$ denotes the red ("R") filter magnitude of an extended comet sampled in a $5^{\prime \prime}-10^{\prime \prime}$ annulus. The apparent magnitude of a point source comet (a bare nucleus) is more simply written $m_{\lambda}$. 


\section{Instrumentation}

Two-dimensional imaging is more or less a necessity in the study of faint comets, since background stars and galaxies severely limit the accuracy of photometry taken with one-dimensional (photomultiplier) detectors (Fig. 1). A wide variety of two-dimensional detectors, including the human retina, the photographic plate, vidicon imagers, electronographs and, most recently, the charge coupled device (CCD), have been applied to the photometry of comets. Of these, the CCD best meets the practical cometary requirements of a linear, two-dimensional detector with high sensitivity over a wide range of wavelengths. Accordingly, we here describe the properties of the $\mathrm{CCD}$ as they are relevant to the photometry of comets.

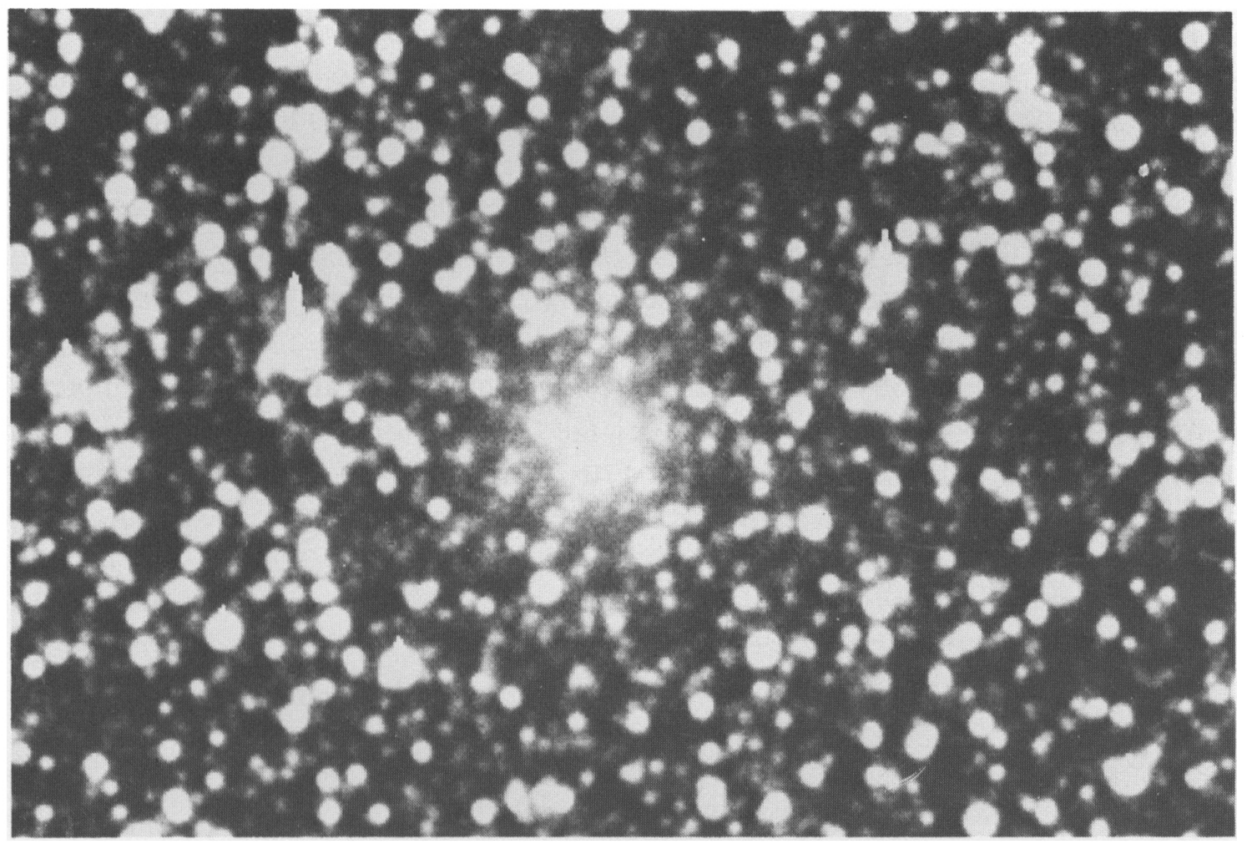

Figure 1. CCD image of comet Bowell taken 1982 September at the Kitt Peak 2.1-m telescope. The image graphically illustrates the need for accurate subtraction of background stars and galaxies in the photometry of comets. Field of view is 4 arcmin square.

The CCD combines the linearity and sensitivity of a photomultiplier with the twodimensional imaging capability of the photographic plate, and it has a wider range of useful wavelengths than either (say, $4000 \AA \leq \lambda \leq 10000 \AA$ for an uncoated device). Two representative CCDs are shown in Fig. 2 (Luppino 1989). A CCD is a centimeterwide wafer of silicon divided into independent pixels, each characterized by a wavelength dependent sensitivity, $s_{i, j}(\lambda)\left[\mathrm{e}^{-} /\left(\mathrm{J} \mathrm{m}^{-2} \mathrm{~Hz}^{-1}\right)\right]$, a dark (thermal) emission rate, 
$d_{i, j}[\mathrm{e} / \mathrm{s}]$, and a bias level, $b_{i, j}\left[\mathrm{e}^{-}\right]$. Devices with $2048 \times 2048$ pixels are now in astronomical use, although rare; CCD's half this size are common. The signal (measured in electrons) in the $i, j$ th pixel is

$$
S_{i, j}(\lambda)=\left(s_{i, j}(\lambda) I_{i, j}(\lambda)+d_{i, j}\right) t+b_{i, j}
$$

where $I_{i, j}(\lambda)\left[\mathrm{W} \mathrm{m} \mathrm{m}^{-2} \mathrm{~Hz}^{-1}\right]$ is the sought-after flux density incident on the $i, j$ th pixel, and $t[\mathrm{~s}]$ is the integration time. For example, the TI-4849 CCD shown in Fig. 2 has $584 \times 390$ pixels, with each pixel being $22-\mu \mathrm{m}$ square. Each pixel can hold a maximum of about $2.5 \times 10^{5}$ electrons -- with larger charges the pixel response "saturates" (i.e., departs dramatically from equation (1)). A prime measure of the quality of a CCD for astronomical imaging is the efficiency with which charge can be transferred from one pixel to the next during readout. Because the charge must be transferred between pixels many times during a single readout, the charge transfer efficiency must be close to unity. For the TI-4849 device, this "charge transfer efficiency" is $99.9996 \%$ (Luppino 1989; c.f. Fort 1985). The relative sensitivity among pixels, which commonly varies by $20 \%$ $30 \%$ across the width of the CCD, is measured by imaging a source of uniform illumination (a "flat field") for which $I_{i, j}(\lambda)=$ constant for all pixels. Useful flat fields include the illuminated interior of the observatory dome, the twilight sky, and medianfiltered images of the night sky itself. The bias level is measured by reading out the CCD in darkness. Dark emission is determined by reading out the chip after a prolonged period during which no photons are allowed to reach the CCD. In general, $d_{i, j}$ is minimized by cooling the CCD to a temperature $T \sim 170 \mathrm{~K}$, and may be neglected for typical integrations of a few hundred seconds. In this case, the equation

$$
I_{i, j}(\lambda)=\frac{S_{i, j}(\lambda)-b_{i, j}}{s_{i, j}(\lambda) t}
$$

gives the flux density incident on the $i, j$ th pixel. Absolute photometric calibration is obtained from observations of standard stars. The peak quantum efficiency occurs generally near $\lambda \sim 7000 \AA$, and is near $70 \%$ in the better devices.

The $\mathrm{CCD}$ is sensitive to cosmic rays as well as to photons. Long integrations must be spatially filtered to remove cosmic rays, as well as defective pixels in which charge transfer may be blocked, leading to dead columns. Reading out the pixels of a CCD introduces "read noise". For the TI-4849 device described above, the read-noise is $\sim 9$ electrons, and is dwarfed by sky noise in most broad-band astronomical exposures. Not all pixels on a CCD faithfully obey eq. (1); several important deviations from linearity are discussed by Baum et al. (1981). In certain CCDs, the effective $s_{i}(\lambda)$ is subject to fringe-like spatial variations due to interference produced by multiple internal reflections 
between the front and back surfaces of the CCD chip. These fringes can sometimes be removed by appropriate flat fielding, but they can be a major problem at $\lambda>0.7 \mu \mathrm{m}$, where the night sky is bright in the lines of atmospheric $\mathrm{OH}$ (e.g., Gunn and Westphal 1981). Some of the steps useful in the processing of CCD images in a cometary context are reviewed by Larson (1986).

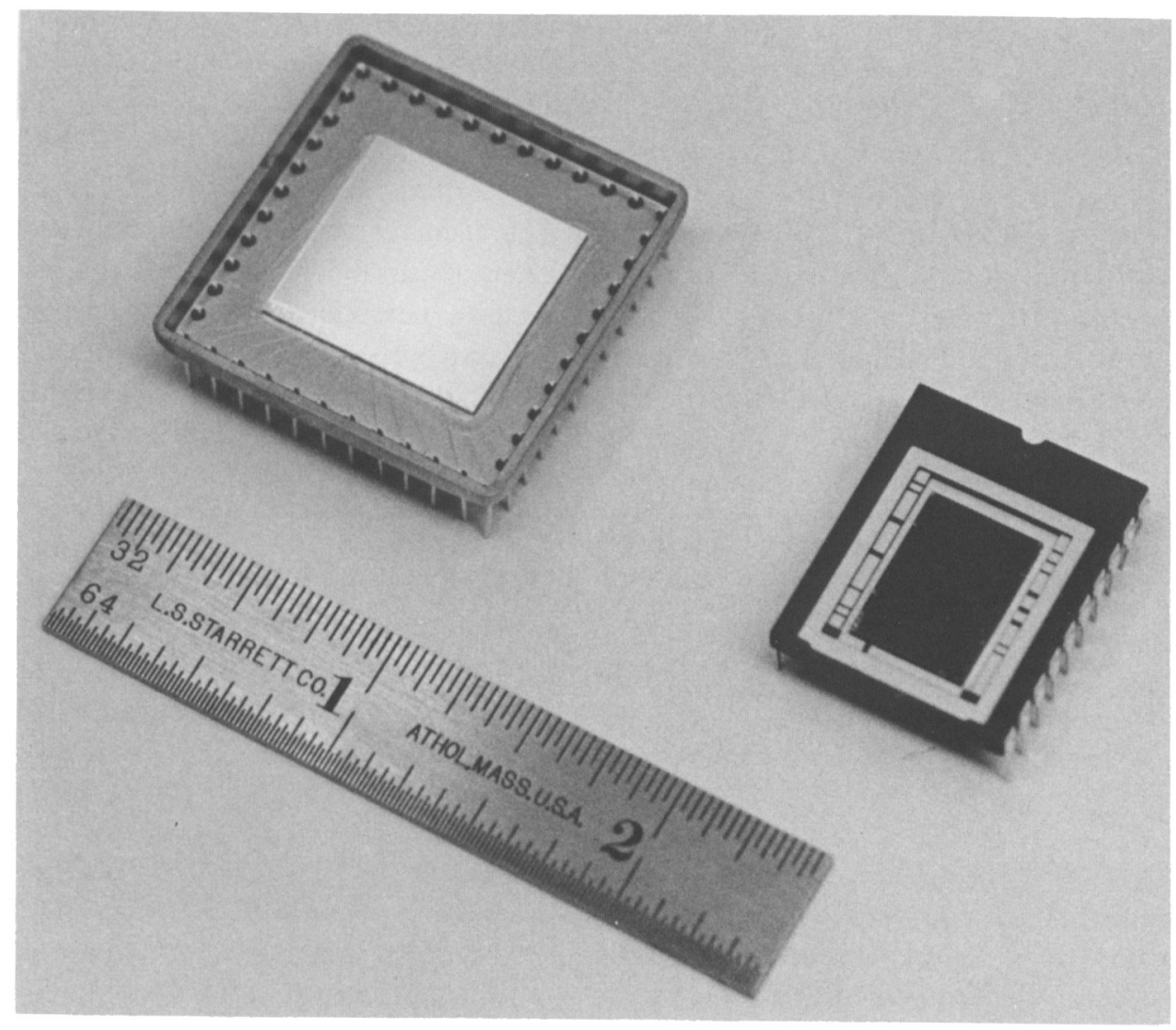

Figure 2. Typical charge coupled devices used for cometary photometry. The larger CCD is a virtual phase TI 4849 with $584 \times 390$ pixels, and the smaller a TI $850 \times 750$. The scale ruler is 2.5 inches in length. Figure courtesy of Gerry Luppino, MIT.

The main disadvantage of CCDs for the study of comets is their small physical size, which limits the field of view. There is still no effective replacement for the photographic plate in wide field (e.g., Schmidt) studies of cometary tails. However, some wide field CCD observations have been attempted (e.g., West et al. 1986; Lamy et al. 1987), and arrays of large-format CCDs mounted in Schmidt or other survey-type cameras can be expected in the near future. 


\section{Photometry of Nuclei}

Photometry of cometary nuclei is conceptually very simple, so I discuss this topic first. The study of nuclei is motivated by the belief that these bodies are chemically and physically primitive, and that they may preserve information from early times in the solar system. Essentially, the problem is to find a comet in which the scattering cross section of the coma is small compared with the cross section of the nucleus. This can be done either by observing comets of intrinsically low activity, or by examining comets at large heliocentric distances, $R$ [AU], where sublimation is minimized by low temperatures. The angle subtended by a $10 \mathrm{~km}$ diameter nucleus at $1 \mathrm{AU}$ is $\sim 0.01$ "; the nuclei are all point sources. The pre-perihelion evolution in the morphology of a single comet (P/Halley) is shown in Fig. 3 -- the growth from a point source object to a fully resolved comet is obvious. Early evidence for the detection of bare nuclei was reviewed by Sekanina (1976). Fay and Wisniewski (1978) reported a measurement of the nucleus of $\mathrm{P} / \mathrm{d}$ 'Arrest using a photomultiplier. Cometary nuclei have been observed photometrically in the optical since the mid-1980's, roughly coinciding with the ready availability of CCDs. While the measurements are still not "routine", it is likely that they will lead to the accumulation of a statistically useful sample of nucleus data within a few years.

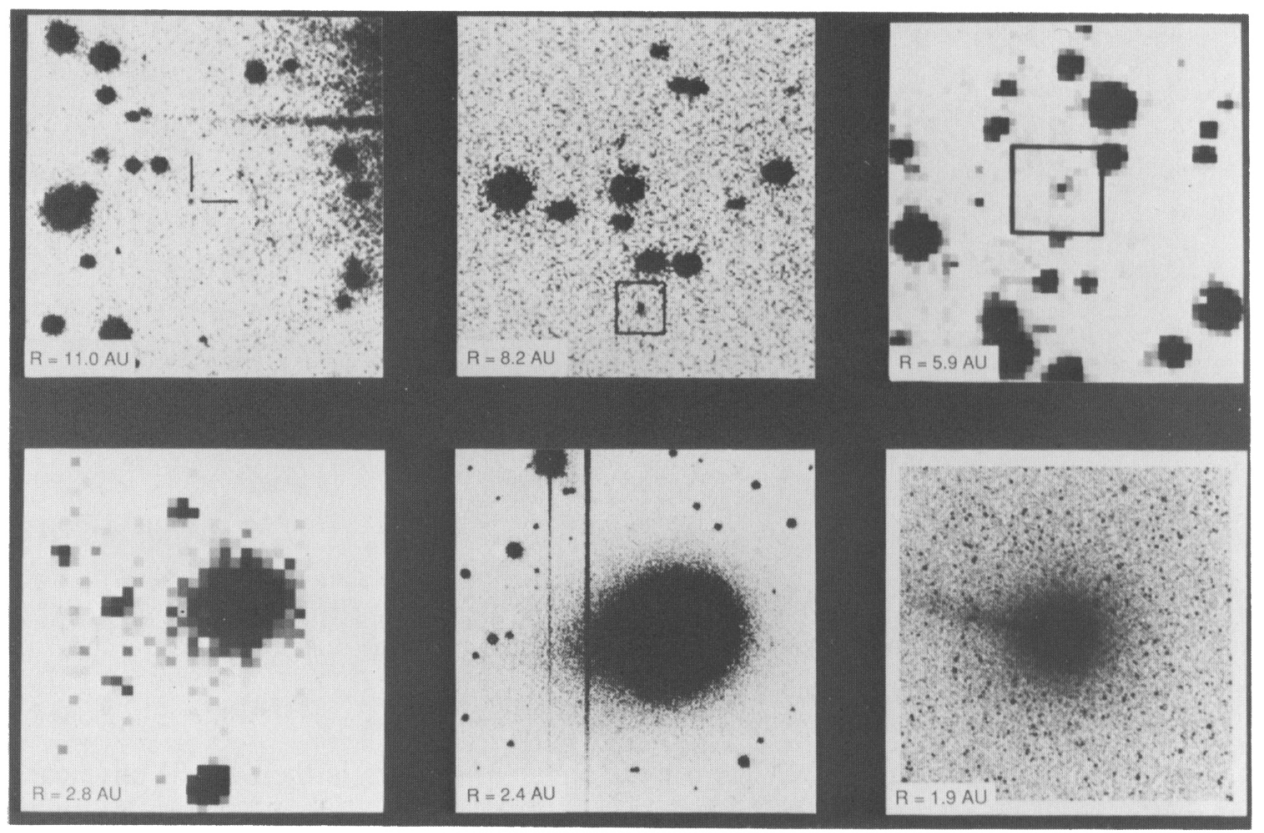

Figure 3. Mosaic of images showing the development of $\mathrm{P} / \mathrm{Halley}$ as a function of heliocentric distance. The heliocentric distances are indicated in the figure. The comet is stellar at $R=11.0$ and $8.2 \mathrm{AU}$, is marginally resolved at $R=5.9 \mathrm{AU}$, and has a well-developed dust coma at all smaller $R$. The image at $R=1.9 \mathrm{AU}$ shows a faint plasma tail extending beyond the inner coma. 
The apparent magnitude of a nucleus, $m_{\lambda}$, is related to its absolute magnitude (the magnitude reduced to unit heliocentric and geocentric distances and to zero degrees phase) $m_{\lambda}(1,1,0)$ by the inverse square law

$$
m_{\lambda}=m_{\lambda}(1,1,0)+5 \log _{10}(R \Delta)+f(\alpha)
$$

where $R$ and $\Delta$ are the heliocentric and geocentric distances in $A U$, and $f(\alpha)$ is a function which accounts for the darkening of the nucleus with increasing phase angle, $\alpha$ [deg.]. A physical model of phase darkening has been advanced (see Bowell et al. 1989 ), and the intricate expression for $f(\alpha)$ derived from this model has recently been adopted as the standard in studies of asteroids. For our present purposes a linear approximation, $f(\alpha)=\beta \alpha$, where $\beta$ [mag. / deg.] is the "phase coefficient", is more convenient. Estimates of the phase coefficient from optical photometry of the nuclei of comets $\mathrm{P} / \mathrm{Neujmin} 1, \mathrm{P} /$ Encke and $\mathrm{P} /$ Tempel 2 fall in the range $0.03 \leq \beta \leq 0.04$ mag. / deg., comparable to the $\beta$ found in low-albedo main-belt asteroids (Bowell et al. 1989). Nuclei studied to date have $m_{R}(1,1, \hat{0}) \sim 13-15$, comparable to the smallest main-belt asteroids for which physical data exist. When observed at $R \sim 4 \mathrm{AU}, \Delta \sim 3$ $\mathrm{AU}$ (to minimize coma), the apparent magnitudes are typically $m_{R} \sim 19-21$. Therefore, telescopes of moderate aperture are needed to study the nuclei of comets at distances large enough to reduce the influence of the coma, and with time resolution ( $\sim 5$ minutes) adequate to sample rotational variations. The main practical problem in nuclear photometry concerns background subtraction. At $m_{R} \geq 19$, the surface density of background objects is high enough that stars and galaxies routinely interfere with nucleus photometry: careful background subtraction is mandatory. (Russell 1916)

The apparent magnitude is related to the physical properties of the nucleus by

$$
g_{\lambda} \Phi(\alpha) C=2.25 \times 10^{22} R^{2} \Delta^{2} \pi 10^{0.4\left(m_{s U N}-m_{\lambda}\right)}
$$

where $g_{\lambda}$ is the geometric albedo at the wavelength of observation, $m_{S U N}$ is the apparent magnitude of the sun, $C$ is the geometric cross section of the nucleus and $\Phi(\alpha)=10^{-0.4 \beta \alpha}$. Optical photometry yields the "optical cross section" $g_{\lambda} \Phi(\alpha) C$. Repeated measurements give the rotational lightcurve; a single example is given in Fig. 4.

Conditions for the detection of a bare nucleus include:

(a). The image should appear unresolved.

(b). The time-averaged absolute magnitude (a measure of the optical cross section of 
the nucleus), measured at different positions in the orbit, should conform to $m_{\lambda}(1,1,0) \sim$ constant.

(c). Repeated measurements of $m_{\lambda}(1,1,0)$ should reveal a lightcurve due to rotational modulation of the scattered light.

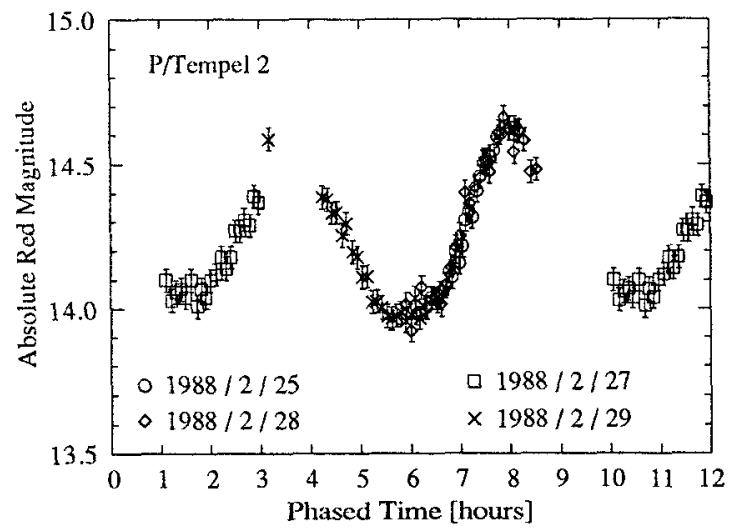

Figure 4. Rotational lightcurve of the nucleus of $\mathrm{P} / \mathrm{Tempel} 2$. Figure from Jewitt and Luu (1989).

Numerous caveats to these conditions exist. For instance, with regard to condition $(a)$, a compact coma may be unresolved and yet still influence or even dominate the photometry of a faint comet. For a standard steady-state coma (see \$4.2), the integrated magnitude, $m_{\lambda}(p)$, within a diaphragm of radius $p["]$, is related to the surface brightness, $\Sigma_{\lambda}(p)$, at radius $p$ by

$$
\Sigma_{\lambda}(p)=m_{\lambda}\left(p^{\prime \prime}\right)+2.5 \log \left(2 \pi p^{2}\right)
$$

(Jewitt and Danielson 1984). For example, with a $p=3^{\prime \prime}$ radius diaphragm, eq. (5) gives $m(p)=\Sigma_{\lambda}-4.38$. In typical CCD data a surface brightness $\Sigma_{R} \sim 26$ mag./arcsec ${ }^{2}$, can just be measured. Therefore, in 1" - 2" seeing, a $m_{R}(p) \sim 21.6$ magnitude comet could be dominated by coma and yet appear unresolved. Thus, condition (a) does not guarantee the absence of a coma in a faint comet. Conversely, several cases exist in which nuclei were measured in the presence of a resolved coma (e.g., P/Tempel 2, A'Hearn et al. 1989; Jewitt and Luu 1989; P/Halley, West and Jorgenson 1989). Condition (b) is strictly valid only for a uniform, spherical nucleus. The projected cross section of a nucleus may change if the nucleus is highly aspherical or has albedo spots. Rotational lightcurves (condition $(c)$ ) may not be observed in spherical or oblate-spheroidal, uniform nuclei, or in nuclei with rotation vectors parallel to the line of sight. Detection of a periodic lightcurve strongly suggests that a nucleus has been observed -- absence of a lightcurve is equivocal in this regard.

In practice, it is rarely simple to decide whether or not a bare nucleus has been 
observed. A stellar comet which shows persistent cyclic variations about a stable mean $m_{\lambda}(1,1,0)$ is probably a bare nucleus. In less compelling instances, a carefully reasoned physical argument must be made for the detection of a nucleus.

The irregular and generally sparse sampling of the nucleus lightcurves published to date precludes any meaningful search for multiple periodicities in the photometry, although multiple periodicities have sometimes been claimed (see Belton 1990). Thus, while the nuclei are widely expected to precess under the action of mass loss torques (e.g., Wilhelm 1986), there is no compelling evidence for precession in existing cometary photometry. Even the rotation period is often poorly constrained by available photometry. In addition, weak activity may corrupt the strict periodicity of the "nucleus" lightcurve so that formal tests of the significance of periodicity may be misleading.

The main results of recent photometry of cometary nuclei are (see also Table 1):

(1) Rotation periods deduced from photometry generally disagree with rotation periods deduced from less direct methods. In particular, the "halo method" of Whipple (1982) does not yield reproducible nucleus rotation periods (Whipple 1989).

(2) The mean nucleus rotation period, $\bar{P}=12.6 \pm 2.3 \mathrm{hrs}$ (comets P/Arend-Rigaux, $\mathrm{P} /$ Neujmin 1, P/Encke and P/Tempel 2), is consistent with the mean main-belt asteroid rotation period $\bar{P} \sim 9$ hrs (Dermott et al. 1984), within the large uncertainties on the two means.

(3) The mean photometric range of the lightcurves of the nuclei is $\Delta m_{R}=0.8 \pm 0.2$ mag., substantially larger than the $\Delta m_{R}=0.2 \pm 0.2$ mag. range found in main-belt asteroids of comparable size (Fig. 5). The most likely explanation is that the nuclei of comets are grossly aspherical compared to their asteroidal counterparts (Jewitt and Meech 1988b). Whether this is a result of irregular mass loss or a primordial effect is not known.

(4) Photometric studies are now being used to test physical models of the nucleus. For example, the "fan-model" of Sekanina (1987) appears consistent with CCD photometry of P/Tempel 2 (Jewitt and Luu 1989), while the precession model of Whipple and Sekanina (1979) is not consistent with CCD photometry of P/Encke (Jewitt and Meech 1987b; Luu and Jewitt 1990).

It is not yet clear which, if any, of the measured properties reveal the characteristics of primordial comets -- the rotations, sizes, shapes and surface optical properties may all have been modified in recent times by mass loss. Continued photometry will hopefully lead to an improved understanding of cometary nuclei and of their possible relationships 


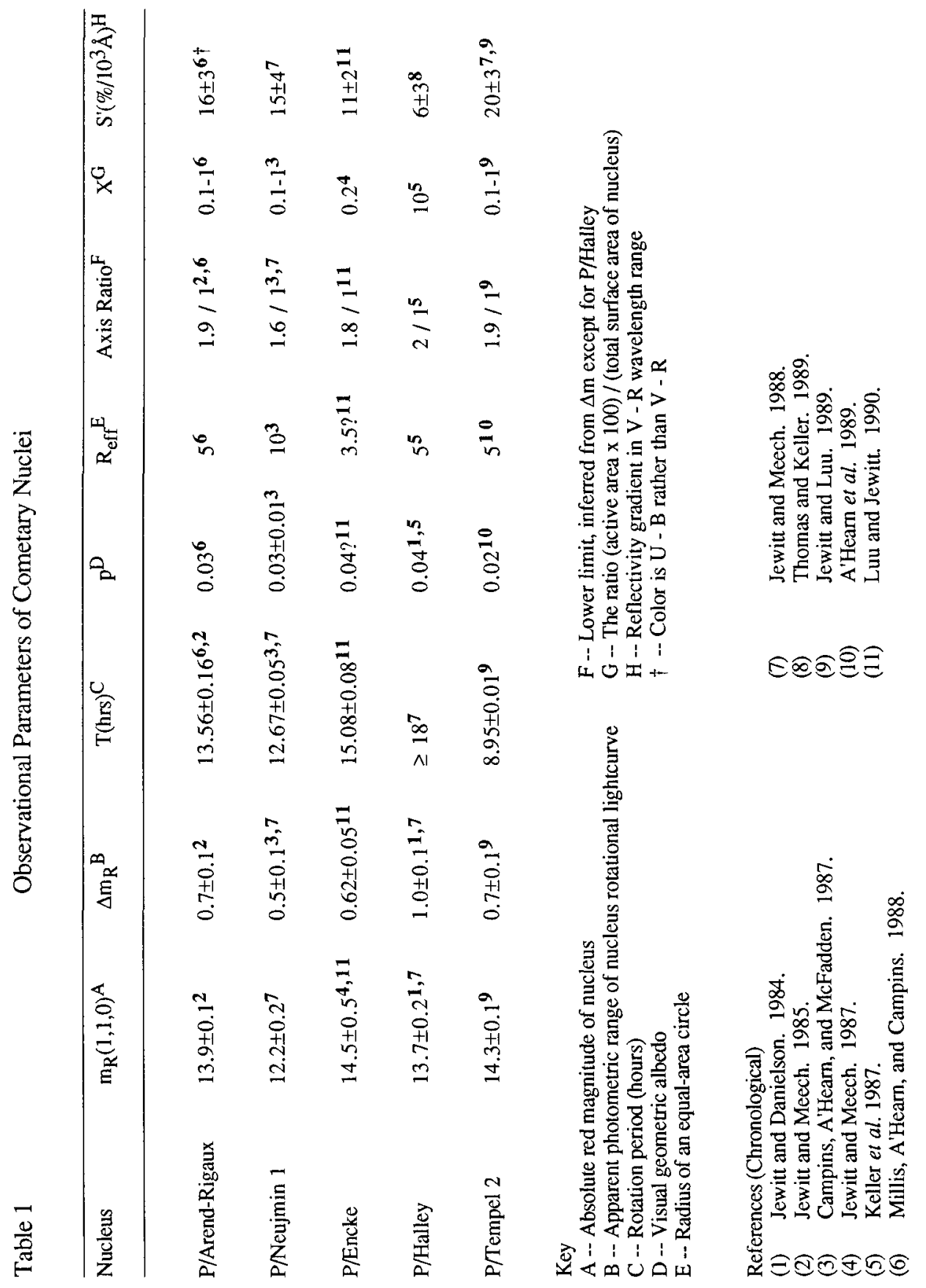


with other small solar-system bodies.

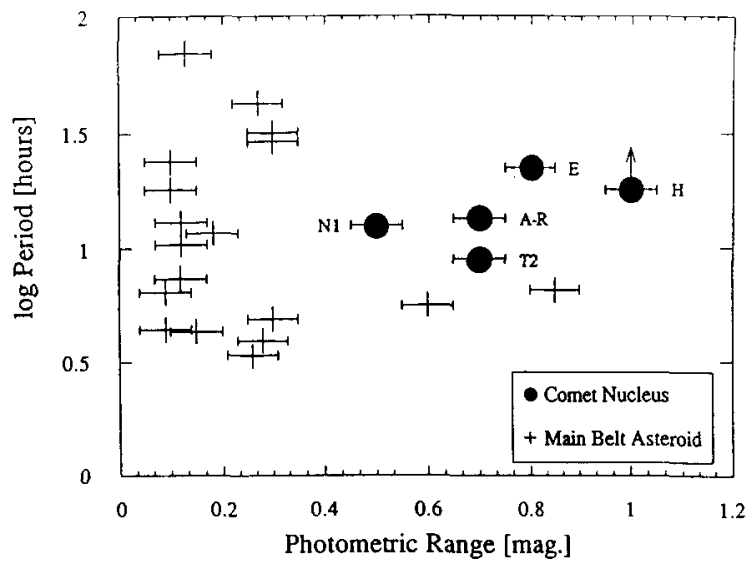

Figure 5. Rotational lightcurve range vs. period for comet nuclei and small main-belt asteroids. Figure adapted from Jewitt and Luu (1989).

\section{Surface Photometry}

\subsection{THE GAS AND THE DUST}

The cometary spectrum consists of solar continuum radiation scattered by the central nucleus (typical radius $\sim 5 \mathrm{~km}$ ) and coma dust grains (optically representative size $a \sim 1 \mu \mathrm{m}$ ), in addition to emission from the neutral and ionized radicals of the gas coma. It is imperative that the continuum and molecular band emissions be separated in cometary photometry, since it is nearly impossible to make physical sense of photometry in which the two components are mixed. A large fraction of all published cometary photometry is devalued because the steps needed to separate the gas from the dust have not been taken. Fortunately, the necessary steps are simple, involving nothing more than the use of appropriate narrow-band filters or the use of a spectrograph. The gaseous emissions are a stronger function of heliocentric distance than the dust continuum, so that even comets at $R \geq 2$ - 3 AU commonly show pure continuum spectra. In these cases, broad-band filters may be used. In the following, I treat the dust and gas components of the cometary coma as distinct. Recent evidence suggests that the physical distinction may not be so clear-cut as is implied; nevertheless, the adopted format has the advantage of clarity.

I first discuss the distribution of the surface brightness, $B_{\lambda}\left[\mathrm{W} \mathrm{m} \mathrm{m}^{-2} \mathrm{~Hz}^{-1} /\right.$ $\left.(\operatorname{arcsec})^{2}\right]$, as a function of radial position on the coma of an active comet. The surface brightness in mag. $/(\operatorname{arcsec})^{2}, \Sigma_{\lambda}$, is related to $B_{\lambda}$ by $\Sigma_{\lambda}=-2.5 \log \left(B_{\lambda}\right)+\kappa$, where $\kappa$ is a constant of the particular magnitude system used. The surface brightness profile provides a useful tool from which certain aspects of the 3D structure of the coma can be deduced. Furthermore, some knowledge of the surface brightness profile is essential if 
any physical interpretation is to be placed on photometry of comets (for instance, in the derivation of production rates), since most such photometry is taken using small apertures which sample only a fraction of the coma. In real comets, the simultaneous action of different physical effects makes the interpretation of the profile a non-trivial matter. The approach I follow is to first examine simplified models of the coma that can later be used to understand major features of the comae of real comets. Specific properties of real comets are discussed in $\$ 4.6$.

\subsection{SURFACE PHOTOMETRY OF DUST}

The line of sight optical depth of the coma is a fundamental quantity which largely determines the appearance of the comet as it is projected into the plane of the sky and which may influence the energy balance at the surface of the nucleus. The scattering optical depth through a spherically symmetric coma is

$$
\tau(p)=\int_{p=\text { constant }} N_{1}(r) Q_{s}(a, \lambda) \pi a^{2} d l
$$

where $N_{1}(r)$ is the particle number density at radial distance $r$ from the nucleus, $Q_{s}(a, \lambda) \pi a^{2}$ is the scattering cross section of a single particle in the coma of radius, $a$, and the integration is carried out along a line of sight, $l$, making a fixed impact parameter, $p$, with the nucleus. (Note, the symbols $r, R$, and $\rho$ are sometimes employed by other authors to denote the impact parameter. All of these symbols have other uses in this review). Equivalent definitions can be given for the absorption and extinction optical depths by replacing the scattering efficiency $Q_{s}(a, \lambda)$ with $Q_{a}(a, \lambda)$ or $Q_{e}(a, \lambda)$.

To evaluate eq. (6), we need information about the spatial variation of the number density, $N_{l}(r)$. To illustrate a particular case (which happens to have physical significance and practical value in comet photometry), I address a spherically symmetric, steady-state coma in which the number density is given by the inverse square law

$$
N_{1}(r)=\frac{1}{4 \pi r^{2} v_{g r}} \frac{d N}{d t} \quad\left(r \geq r_{n}\right)
$$

where $d N / d t$ is the rate of production of grains from the nucleus. (Note, the symbol $Q$ is sometimes employed by other authors to denote the production rate, while, to confuse matters further, the column density is often written $N$ ). Integration of eq. (6) then gives 


$$
\tau(0)=\frac{Q_{s}(a, \lambda) a^{2}}{4 v_{g r} r_{n}} \frac{d N}{d t}
$$

for the optical depth measured along the line connecting the centers of the sun and the nucleus. In eqs. (7) and (8), $v_{g r}$ is the radial velocity of outflow of the particles from the nucleus, and $r_{n}$ is the radius of the (assumed) spherical nucleus.

Integration of eq. (6) along a line of sight, which makes impact parameter $p \geq r_{n}$, gives

$$
\tau(p)=\frac{\pi r_{n} \tau(0)}{p}
$$

A simple thought experiment demonstrates that the coma must be optically thin, $\tau(0) \leq 1$, along the sun-comet line, even in comets which are very close to the sun and very active. Consider a comet in which the coma is optically thick, $\tau(0)>1$. The solar radiation impinging on the nucleus will be attenuated by a factor $\sim \mathrm{e}^{-\tau(0)}$, causing a reduction in the heating rate and in the temperature of the sublimating surface. Since the sublimation rate is a very strong function of the temperature, the sublimation will drop precipitously, leading to a net reduction in the mass in the coma along the sun-comet line, killing the source of the high optical depth. The optical depth will stabilize at about unity, $\tau(0) \sim 1$, provided there is sufficient mass loss to sustain this optical depth. The essential correctness of this very simple argument is shown both by detailed numerical calculations (Hellmich and Keller 1981; Weissman and Keiffer 1982), which show that $\tau(0) \sim 1$ for comet P/Halley near perihelion, and by observations (Ney 1982; Keller et al. 1987). Attenuation of radiation incident on the day-side of the nucleus may be partially counterbalanced by scattering of radiation from the coma onto the night-side. The net radiative effects of a $\tau(0) \sim 1$ coma on the nucleus sublimation rate are clearly described by Salo (1988). In most comets, the mass loss is too small to sustain $\tau(0) \sim 1$, even at perihelion, and the solar radiation reaches the day side of the nucleus unimpeded, while scattering from the coma contributes only slightly to heating the night side. Of course, the real case is not quite so simple as I suggest, since the coma may be optically thick at certain wavelengths (e.g., the resonant wavelengths of abundant coma molecules) and yet still satisfy the condition $\tau(0) \leq 1$ when an average over all wavelengths is considered. It is also possible that large optical depths may be attained briefly during outbursts and other non-steady-state events. Evidence that the coma may sometimes be optically thick (in extinction) is limited to a few unconfirmed reports of the dimming of stars during appulses (e.g., Larson and A'Hearn 1984; Dollfus and Suchail 1987).

Given that $\tau(0) \leq 1$, and more usually, $\tau(0) \ll 1$, it is clear from eq. (9) that the 
coma must be everywhere optically thin $\left(\tau(p)<<1\right.$ for all $\left.p>>r_{n}\right)$. This result is very pleasing, because it means that photometric models of the coma can be simple (multiple scattering need not be considered), and yet still can represent the coma with considerable accuracy. In the optically thin case, the surface brightness of the coma at a given impact parameter is (O'Dell 1971)

$$
B_{\lambda}(p)=\frac{F_{S U N} \tau(p)}{4 \pi}
$$

where $F_{S U N}\left[\mathrm{~W} \mathrm{~m} \mathrm{~m}^{-2} \mathrm{~Hz}^{-1}\right.$ ] is the solar flux density incident on the coma. It is immediately clear from eqs. (9) and (10) that the surface brightness of the coma in the spherically symmetric, steady-state case follows the simple law

$$
B_{\lambda}(p)=\frac{K}{p}
$$

where $K$ is a constant. Photometric observations confirm that the inner coma radial surface brightness profiles of many comets are well described by eq. (11) (see Fig. 6; Jewitt and Meech 1987a; Baum and Kreidl 1986; Gammelgaard and Thompson 1988). Hence, it appears that many dust comae can be described by the spherically symmetric, steady-state coma model and thus obey eq. (11), but this is not always the case, as we now discuss.

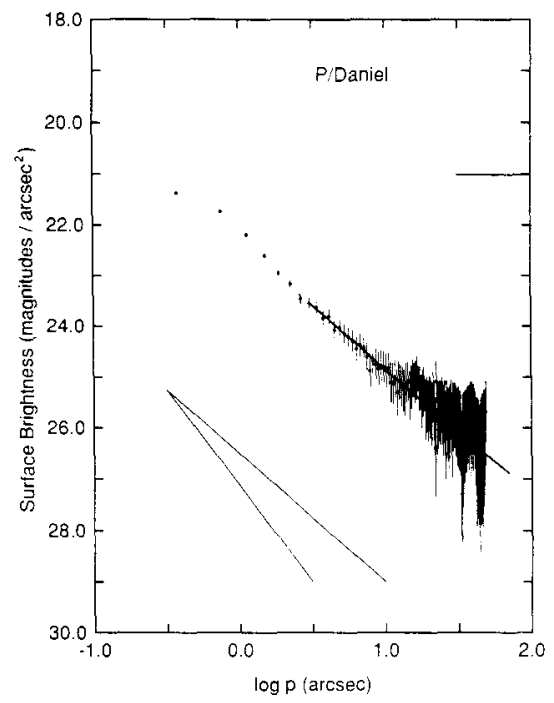

Figure 6. Surface brightness profile of $P /$ Daniel. The gradient of the profile is consistent with $m=-1$. The straight lines indicate $m=-1$ and $m=-1.5$. The line through the data has $m$ $=-1$. Figure from Jewitt and Meech (1987a).

\subsection{EFFECT OF RADIATION PRESSURE}

The spherically symmetric, steady-state coma model must be augmented if it is to 
represent the surface brightness profiles of real comets, especially at large $p$, where eq. (11) breaks down. The most important effect neglected so far is solar radiation pressure (Eddington 1910), which restricts the extent of the coma in the sunward direction to a length scale

$$
X_{R}=\frac{v_{g r}^{2} R^{2}}{2 \beta g_{s u n}(1)}
$$

where $v_{g r}$ is the grain terminal velocity, $R$ [AU] is the heliocentric distance, $\beta g_{s u n}(1) / R^{2}$ is the acceleration of the grain due to solar radiation pressure and $g_{\text {sun }}(1)$ $=6 \times 10^{-3} \mathrm{~m} \mathrm{~s}^{-2}$ is the solar gravity at $R=1 \mathrm{AU}$. The radiation pressure factor, $\beta$, is a function of the size, shape, composition and density of the grain (van de Hulst 1957). It has been computed for spherical, Mie scatterers of cometary interest by Burns et al. (1979). The grain velocity in eq. (12) is a function of the grain size, and of the gas flux emanating from the nucleus. Physically, the terminal velocity may be estimated by equating the momentum of the grain to the total momentum delivered by gas molecules striking the grain within a few nucleus radii of its source (the gas at larger distances is very dilute). Small grains (radius $a \leq 1 \mu \mathrm{m}$ ) in an active comet are expected to be dynamically well-coupled to the gas, and should have terminal velocity comparable to the gas flow speed. Conversely, large grains can be accelerated only to a terminal velocity small compared to the gas flow speed (c.f. Gombosi 1986; Vaisberg et al. 1987). As a first approximation, I assume that the latter speed is approximately given by the Bobrovnikoff-Delsemme relation (Delsemme 1982)

$$
v_{g r} \sim 580 R^{-0.5}
$$

So that, for a comet at $R=1 \mathrm{AU}$, in which $\beta=1$, the length scale is $X_{R} \sim 3 \times 10^{4} \mathrm{~km}$, subtending an angle $\theta \sim 40^{\prime \prime}$ at $\Delta=1$ AU. This explains immediately why most comets fit within the (few sq. arcmin) fields of view of CCD cameras. Notice from eq. (12) and (13) that $X_{R} \propto R$; contrary to our naïve expectations, the grain coma dimension in the sunward direction should actually increase with distance from the sun, and the angle subtended $\left(\theta \propto X_{R} / \Delta\right)$ should be nearly constant with respect to $R$, for $R \gg \Delta$. This, too, is in general accord with observations at a wide range of distances.

Isophote models of simple comae influenced by solar radiation pressure appeared in a prescient work by Wallace and Miller (1958). These authors also investigated the change in the shape of the isophotes due to anisotropy in the ejection of grains from the nucleus (although to keep the problem tractable, they were forced to use a single grain velocity rather than a more realistic velocity distribution). The trajectories of ejected grains are influenced by solar gravity as well as by radiation. Once clear of the nucleus, 
the grains move in independent orbits about the sun, producing the familiar curvature of the dust tail. The shapes of dust tails are described in a model by Finson and Probstein (1968). The fields of view of most CCD cameras are sufficiently small that the curvature due to solar gravity may be neglected in a first examination of the surface brightness of the inner coma. The Finson and Probstein analysis has been applied to wide-field photographic images, and then only rarely.

For practical reasons, it is convenient to measure the surface brightness profile of the coma using a set of concentric, circular annuli. I describe the resultant surface brightness profiles in terms of the logarithmic derivative of the profile at radius $p$

$$
m=\frac{d \ln B(p)}{d \ln p}
$$

Note that $m=-1$ for the simple, spherically symmetric, steady-state coma discussed above (eq. (11)). The effect of radiation pressure is to steepen the coma (decrease $m$ ) with increasing $p$; this is clearly shown in Figs. 7 and 8 . Fig. 8 shows the effect of changing the scale length $X_{R}$ on a Monte Carlo model coma. Careful measurements of profiles like those in Fig. 8 show that the logarithmic gradient $m \rightarrow-1$ for $p<<X_{R}$ and $m \rightarrow-1.5$ as $p \gg X_{R}$ (Jewitt and Meech 1987a). Physically, in the inner coma, the radiation pressure has not had time to deflect the comet grains from their radial paths out from the nucleus and so the spherically symmetric model applies. In the outer coma, the constant acceleration of the grains produces the $m=-1.5$ gradient. The "knee" in the profile occurs at radial distance $p \sim X_{R}$ (eq. (12)).

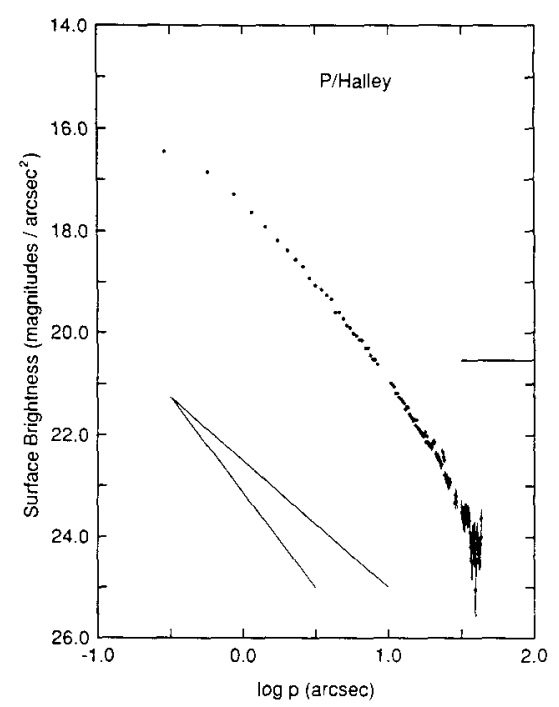

Figure 7. Surface brightness profile of $\mathrm{P} /$ Halley averaged over all azimuth angles. The surface brightness gradient is steeper than can be accounted for by radiation pressure. Fading grains may be responsible for the steep outer profile. The straight lines indicate $m=-1$ and $m=-1.5$. 


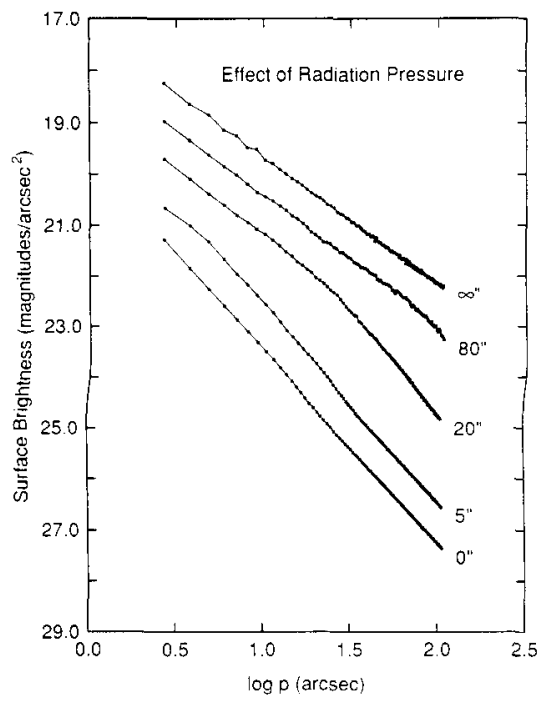

Figure 8. Surface brightness profiles computed from a Monte Carlo model of the cometary coma. The figure shows the effect of varying $X_{R}$ on the surface brightness profile, when viewed at $90^{\circ}$ phase angle. The profiles are azimuthal averages. Figure from Jewitt and Meech (1987a).

The shape of the radiation-pressure distorted coma profile (Fig. 9) is also a function of the phase angle. The models plotted in Fig. 9 show the change in the isophotes of a model dust comet as a function of phase angle. In the zero phase case (Fig $9 \mathrm{a})$, the coma is viewed along its axis of symmetry, parallel to the direction of solar radiation pressure. Therefore, the motion of the grains in the plane of the sky is unaccelerated, analogous to the model described by eq. (11), and the isophotes are concentric circles. At successively larger phases, the asymmetry in the coma due to sunward-hemispheric ejection becomes increasingly prominent. The significance of this profile-phase dependence is that we must compute surface brightness models for the specific $R, \Delta, \alpha$ of individual comets -- generic profiles will not do.

Measurements of the surface brightness profiles of comets can be done accurately using CCDs. There are two practical difficulties. At small $p$, the profile of the comet is influenced by seeing and may be affected by cometary motion with respect to reference stars. At large $p$, the uncertainties due to sky subtraction and imperfect flattening of the $\mathrm{CCD}$ are more important. For comparison with photometric models, the middle radius of the $i$ th annulus, $p_{i}$, must be selected so that the surface brightness at $p=p_{i}$ equals the mean surface brightness within the annulus. With this condition, the effective annulus radii are given by

$$
p_{i}=\left[\frac{2 \int_{p_{1}}^{p_{2}} p^{1+m} d p}{p_{2}^{2}-p_{1}^{2}}\right]^{1 / m}
$$


where $p_{1}$ and $p_{2}$ are the inner and outer radii of the $i$ th annulus. For the $m=-1$ case (eq. (11)), the effective radius is simply $p_{i}=\left(p_{1}+p_{2}\right) / 2$, but for other comae (especially with $m<-1$ ) the difference between the effective radius (eq. (15)) and the average radius can be significant. Unless $m$ is known beforehand, the calculation of the $p_{i}$ must be done iteratively.
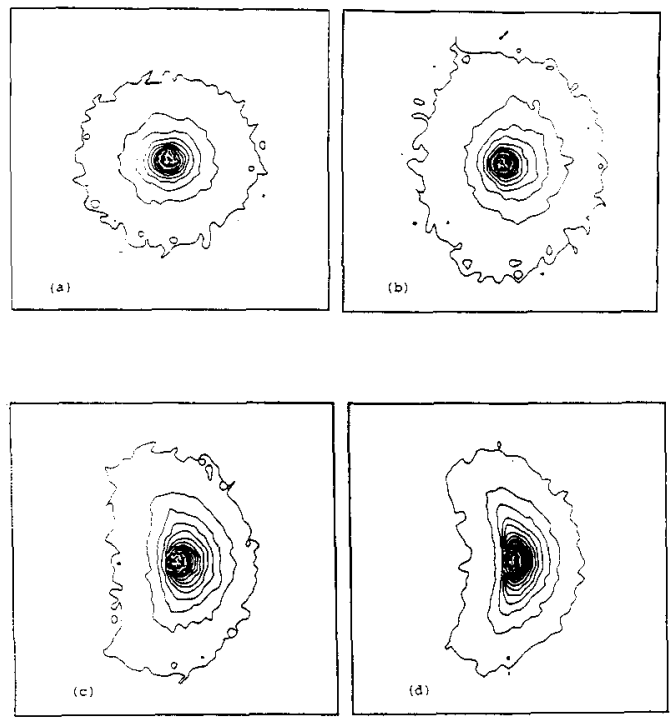

Figure 9. Isophotes of a model comet as a function of phase angle, $\alpha$. The model incorporates a hemispheric (sunward) source of dust grains, described by a power law size distribution (index $q=3$ ). The grains are emitted from the nucleus according to the radiusvelocity relation of Gombosi (1987). The phase angles are (a) $\alpha=0^{\circ}$, (b) $\alpha=30^{\circ}$, (c) $\alpha=60^{\circ}$, (d) $\alpha=90^{\circ}$. In each figure, the Sun is to the right. Regions are $7 \times 10^{7} \mathrm{~m}$ on a side.

It is found that a majority of the surface brightness profiles of comets can be described either by eq. (11) or by models that account for radiation pressure (Jewitt and Meech 1987a; see also Massonne et al. 1986). Thus, on the basis of the surface brightness profiles, we may conclude that the structure of the coma is dominated by the geometrical dilution of the expanding coma (giving rise to the $1 / p(m=-1)$ coma) and by the radiation-pressure-induced acceleration of solid grains (causing distortion of the $1 / p$ coma). Some evidence exists that the continuum surface brightness gradients in some comae may be less steep than $m=-1$. O'Dell et al. (1988), for instance, find $m \sim$ $-0.94 \pm 0.07$ in the inner coma of $\mathrm{P} /$ Halley. These flat profiles are a possible consequence of the time-variable emission from the nucleus $(\$ 6)$.

\subsection{FADING GRAINS}

Baum and Kreidl (1986) reported that a majority of coma profiles are steeper than the canonical $m=-1$. They suggested that fading grains were responsible for the steep profiles, but the neglect of radiation pressure in their analysis leaves room for other interpretations. Jewitt and Meech (1987a) observed that 7 out of 10 surface brightness 
profiles could be fitted by simple models incorporating radiation pressure distortion as described in $\$ 4.3$. However, the outer profiles of the remaining 3 comets were too steep to be fitted by the radiation pressure model, implying that additional processes, including fading grains, may operate. At least two explanations for the steep profiles are plausible: (1) temporal variations in the rate of mass release into the coma (producing a set of waves in the surface brightness profile) and (2) fading grains. Recently, a relatively firm detection of fading grains was achieved in comet P/Tempel 2 (Jewitt and Luu 1989). Whether or not fading grains are common in comets is presently controversial.

The fading grain hypothesis has its origin in the work of Delsemme and Miller (1971), who, using laboratory simulations of the cometary ices, proposed that part of the gaseous coma is liberated by sublimation of an extended water "icy grain halo" (IGH) about the nuclei of certain comets. The surface brightness profile of an IGH, in which grains sublimate from an initial radius $a=a_{0}$ to $a=0$ in time $t_{s u b}$, at a uniform, linear rate $d a / d t=a_{0} / t_{s u b}$, is given by (Delsemme and Miller 1971)

$$
B(x)=K\left[\frac{\operatorname{arcos} x}{x}-2 \ln \frac{1+\left(1-x^{2}\right)^{0.5}}{x}+\left(1-x^{2}\right)^{0.5}\right]
$$

where $K$ is a constant, $x=p / p_{0}, p$ is the usual impact parameter and $p_{0}=v_{g r} t_{s u b}$ is the maximum radial extent of the IGH. Radiation pressure is neglected in eq. (16), as are the distributions of grain size and velocity. $B(x)$ is a sharply truncated function, in which $m \rightarrow-\infty$ as $p \rightarrow p_{0}$. The estimates of $p_{0}$ by Delsemme and Miller were $p_{0} \sim$ $(1-2) \times 10^{4} \mathrm{~km}$ at $R \sim 1 \mathrm{AU}$, comparable to $X_{R}$ (eq. 12). However, more recent arguments suggest that the ice albedos adopted by Delsemme and Miller were too high, and that the reduced albedos and higher temperatures of the ice grains will lead to a much reduced $p_{0}$ (Hanner 1981; Mukai et al. 1985). The current best estimates give $p_{0} \sim 10^{2}-10^{3} \mathrm{~km}$ at $R=1 \mathrm{AU}$ (i.e., $p_{0} \ll X_{R}$ ), comparable to, or smaller than, the typical resolution obtained at a ground based observing site. Thus, water IGHs must be sought in the deconvolved central surface brightness profiles of nearby comets. To date, no such observations have been attempted.

The idea of a distributed source of molecules in the coma persists, and a growing body of evidence suggests that materials having larger sublimation energies and longer fading scale lengths than $\mathrm{H}_{2} \mathrm{O}$ may be present in cometary comae. The $\mathrm{CN}$ and $\mathrm{C}_{2}$ coma jets detected in $\mathrm{P} / \mathrm{Halley}$ have been interpreted as evidence for progressive destruction of solid grains in the coma (A'Hearn et al. 1986a and 1986b; see $\$ 4.6$ ). The $\mathrm{CN}$ and $\mathrm{C}_{2}$ would be released from sub-micron grains, probably by sublimation under solar heating (but see also Combi 1987). Small grains of organic material ("tholin") may attain sublimation temperatures $(T \sim 1000 \mathrm{~K}$ ) even at $R=1 \mathrm{AU}$ (Wallis, Rabilizirov, and 
Wickramasinghe 1987; Lamy and Perrin 1988), and would have sublimation length scales $\sim 10^{4}-10^{5} \mathrm{~km}$. Coincidentally, impact counters on the Giotto probe at Halley detected temporally correlated bursts of dust grain impacts which are best explained as co-moving swarms of fragments from a ruptured parent particle (Simpson et al. 1986). Striae in the dust tails of certain comets have been interpreted as products of grain fragmentation (Sekanina and Farrell 1982). The mechanism causing rupture is not known. Electrostatic explosions have been suggested (Boehnhardt and Fechtig 1987; Notni and Tiersch 1987), although it is perhaps more likely that sublimation of organic binding material within parent grains liberates small, refractory "daughter" grains from larger "parent" grains (c.f. Lamy and Perrin 1988). This parent-daughter relationship among the grains and the gas highlights the similarity between processes acting on the grains and processes acting on cometary gas molecules.

An example of a dust coma profile too steep to be shaped by radiation pressure alone is shown in Fig. 10 (P/Tempel 2). The illustrated convex profile was observed to be stable over a period of months (i.e., the coma profile is known to be in steady state). Figure 10 shows the fit of a fading grain model, in which the time scale of the fading is $10^{5} \mathrm{~s}$. The fading grain model is more sophisticated than the analytic expression given above (eq. (16)), in that grain fading and radiation pressure and grain size and velocity distributions are simultaneously included in the calculation of the profile. It is apparent that the steep outer profile of the comet can be closely matched by the fading grains model. More work is needed to determine whether fading grains are common to all comets, and to estimate the fraction of the gaseous species which are supplied from grains, rather than by sublimation from the nucleus. These questions may be answered by high signal-to-noise ratio surface photometry of the outer portions of comae $\left(p \sim p_{0}\right)$, where the differences between pure radiation pressure and pure fading grain profiles are most prominent.

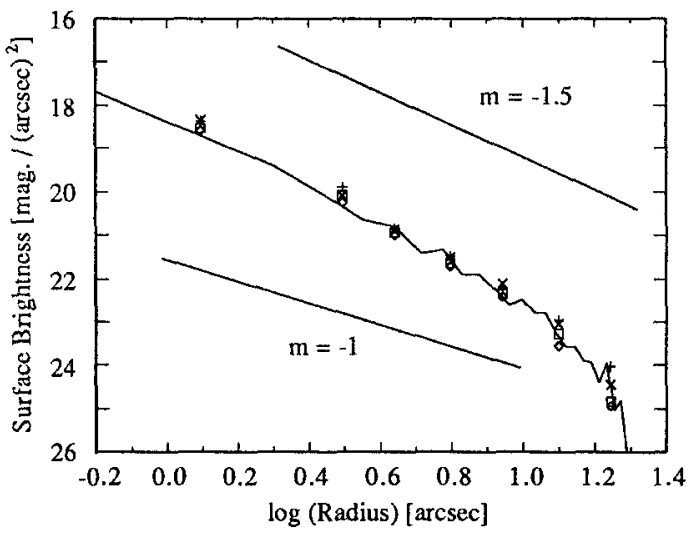

Figure 10. Surface brightness profile of P/Tempel 2. The surface brightness gradient is steeper than can be accounted for by radiation pressure. Fading grains may be responsible for the steep outer profile. Figure from Jewitt and Luu (1989). 
In summary, the dust coma surface brightness profile is determined by a number of physical processes. Careful study of the surface brightness profile can yield important information about these processes. Radiation pressure and fading grains can both produce profiles steeper than $m=-1$. Presumably, both processes act simultaneously to shape the profiles of some comets (e.g., Fig. 10). Profiles steeper than $m=-1.5$ are predicted by fading grain models, but are not found in radiation pressure models examined to date.

\subsection{SURFACE PHOTOMETRY OF GAS}

At small $R$, the gaseous species in comets are responsible for a large fraction of the total scattered radiation. An informative review of the physics of the gas coma has been given by Mendis and Ip (1976). The gaseous species are visible via resonance fluorescence, except for emission from [OI] $6300 \AA, 6363 \AA$, which emits by spontaneous decay from the ${ }^{1} \mathrm{D}$ state in which it is created following the photodissociation of $\mathrm{H}_{2} \mathrm{O}$ (Festou and Feldman 1981). At visible wavelengths, the gas coma is optically thin, so that multiple scattering can be neglected, just as in the dust coma. The simplest and most widely used optically thin gas model is undoubtedly that by Haser (1957). Although important physics is missing from this model, Haser's profiles can be viewed as useful functions with which to fit the surface brightness profiles of gas comae. The model is commonly used to estimate the total number of emitting species from spatially restricted photometry of a small fraction of the coma.

In the Haser model, the observed radicals are fragments ("daughters") of parent molecules ejected from the nucleus along radial lines. The daughter molecules continue to travel radially from the nucleus, until they too are destroyed by photodissociation (producing "granddaughters"). The gas analog of eq. (7), for the number density of daughter molecules at radial distance, $r$, is

$$
N_{d}(r)=\frac{d N_{p} / d t}{4 \pi r^{2} v}\left(\frac{L_{d}}{L_{p}-L_{d}}\right)\left[\exp \left(-\frac{r}{L_{p}}\right)-\exp \left(-\frac{r}{L_{d}}\right)\right]
$$

where $L_{p}$ and $L_{d}$ are the characteristic decay lengths of the parent and daughter species, $d N_{p} / d t$ is the parent molecule production rate, and $r$ is the radial distance from the nucleus. The exponentials in eq. (17) account for random decay of the parent and daughter particles. The column density of daughters at impact parameter $p$ is given by the integral of eq. (17) along the line of sight, in analogy with eq. (8). 


$$
C_{g a s}(p)=\frac{d N_{p l d t}}{2 \pi v p}\left(\frac{L_{d}}{L_{p}-L_{d}}\right) \int_{0}^{\pi / 2}\left[\exp \left(-\frac{p \sec \theta}{L_{p}}\right)-\exp \left(-\frac{p \sec \theta}{L_{d}}\right)\right] d \theta
$$

Since the gas coma is optically thin, the column density and the monochromatic surface brightness are directly proportional. Therefore, eq. (18) gives the shape of the Haser model surface brightness profile, which can be fitted to an observed molecular profile (from a slit spectrum or a narrow-band filter image) to yield $L_{p}$ and $L_{d}$ (if the gas velocity $v$ is known). Notice that the solutions for $L_{p}$ and $L_{d}$ are degenerate (see the amusing exchange on this topic between Delsemme and Malaise in Delsemme 1976). An example of a gas profile is shown in Fig. 11.

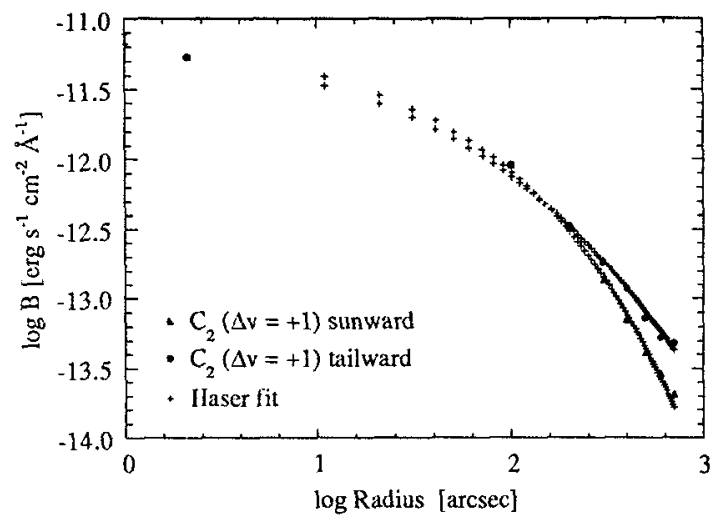

Figure 11. The $\mathrm{C}_{2}$ profile of comet Halley, measured using an IIDS at the Kitt Peak 2.1-m telescope. The difference between the sunward and tailward profiles is the result of radiation pressure. Figure is from Luu (1988).

Values of $L_{p}$ and $L_{d}$ for a range of species are given in A'Hearn (1982), Cochran (1985), and Newburn and Spinrad (1989). The experimental details of photometry and spectrophotometry of cometary gas are well discussed in these works, and will not be repeated here. Scale lengths by the above and other investigators commonly disagree by factors of $2-3$, sometimes by an order of magnitude, even after correction to $R=1 \mathrm{AU}$. This disagreement is not particularly surprising, since the Haser model is based on several simplistic assumptions not likely to be realized in comets. Most notably, the Haser model assumes radial propagation of the parent and daughter species from a point-source nucleus, and a single parent for each daughter species. In reality, collisions will randomize the velocities of the parents within a $10^{4}-\mathrm{km}$ zone about the nucleus, while the daughter molecules are emitted from their parents isotropically, not radially to the nucleus. The main value of the Haser model is in the calculation of production rates of gaseous species from flux density measurements. The degree of uncertainty introduced into the production rates by the use of the Haser model depends on the angular size of the photometry diaphragm compared to the angle subtended by the scale length of the 
observed gas. The production rate uncertainty can be minimized by using a narrow-band imaging detector behind a focal-reducer (e.g., Rees et al. 1986). Unfortunately, relatively little narrow-band imaging of gas comae has been published to date.

In recent times, numerical models have superceded the analytic Haser approach to the interpretation of gas coma photometry. Numerical models of spectacular power and generality have been advanced in a series of papers by Combi and Smyth (1988 and references therein). The "vectorial" model of Festou (1981) has also gained popularity. The Monte Carlo models relax the assumption of purely radial propagation assumed in the Haser paradigm, and they also account for the asymmetry induced in the gas coma by solar radiation pressure (c.f. Fig. 11; note that Haser (1966) has adapted his model to incorporate solar radiation pressure, but the adaptation seems to have been used infrequently). At the present time, with few exceptions, the sophistication of the models is probably not matched by the quality or quantity of the two-dimensional monochromatic imaging data. Several aspects of the gas coma could be investigated using high quality CCD images at molecular band wavelengths. For instance, a given species may have a distributed (coma) source rather than a pure nuclear origin (e.g., Meredith et al. 1989), and the source strength may vary with time. The source function describing the angular distribution of the parent emission at the nucleus can be included in the model, as can a distributed source, and temporal variability. Carefully obtained mono-chromatic images could be used to constrain all of the above source parameters, and so to define the (no doubt) intricate connections between the nucleus morphology and the coma structure. In principle, a numerical model can incorporate any number of subtleties -- the difficulty is to obtain observational material good enough to permit unique model solutions to be discerned. The original Haser model is still used to derive production rates in comets (often from fluxes measured using one dimensional detectors), but the future elucidation of the structure of the gas coma will depend on more complex models applied to two dimensional images of the gas.

\subsection{REAL COMAE}

Ultimately, we wish to understand not just the one-dimensional surface brightness profile, but the full two-dimensional morphology of the coma as measured in an image. To reach this level of understanding, one needs a three-dimensional model of the coma that yields the observed distribution of surface brightness when projected onto the plane of the sky. Unfortunately, the full three-dimensional form of the coma cannot be uniquely inferred from its two-dimensional projection onto the plane of the sky. Moreover, the comae of some comets may exhibit structural variability on timescales of hours or days, so that none of the structures observed in these comae can be assumed to be in true steady state. We mostly possess "snap-shots" of comets, when we really need complete movies. 


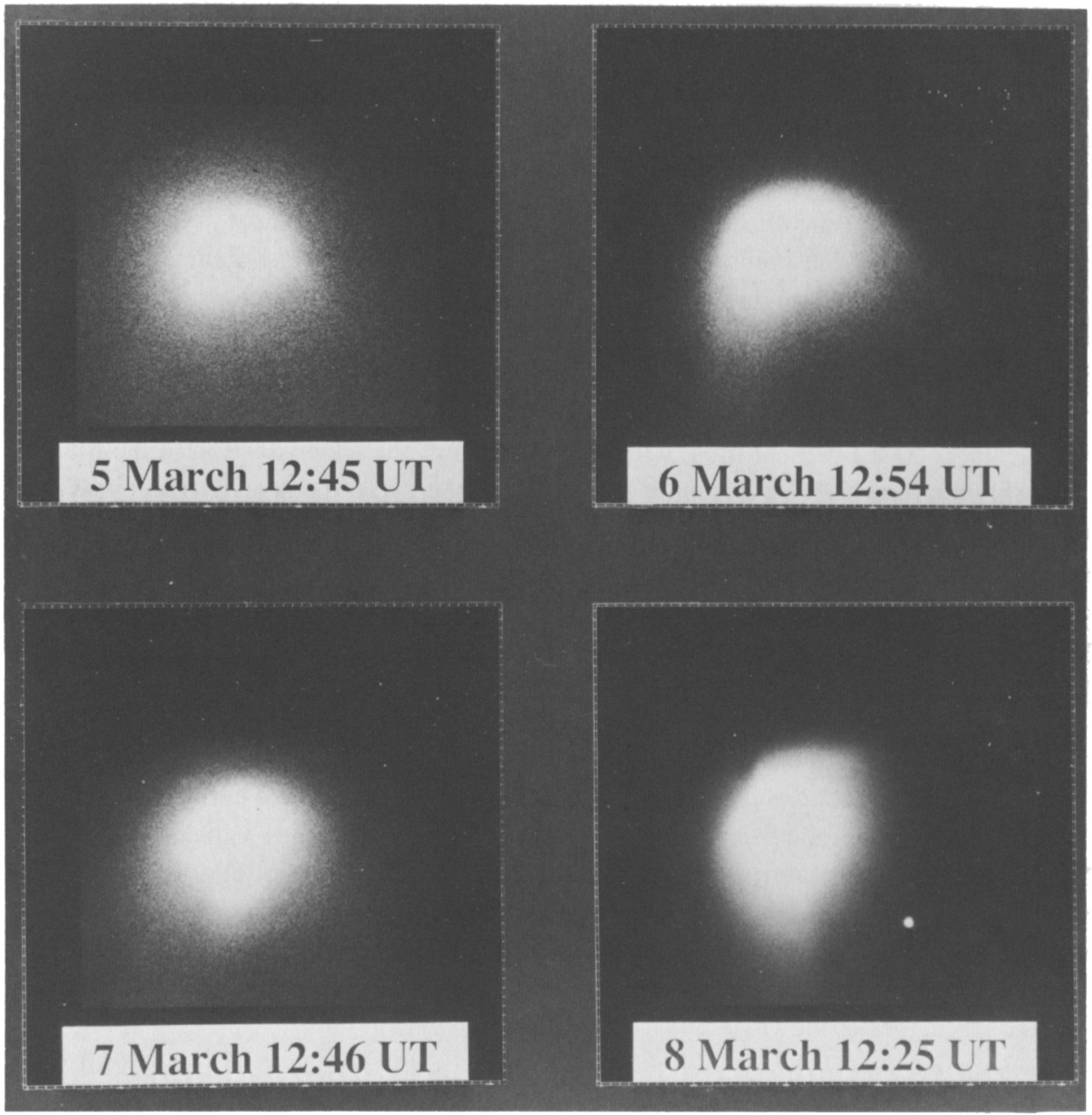

Figure 12. Mosaic of CCD images of P/Halley taken on four consecutive nights in $1986 \mathrm{March}$. The images were taken in the red continuum with integrations of $\sim 5 \mathrm{sec}$. at the Kitt Peak 2.1-m telescope. Each image is $\sim 1$ arcminute on a side. Some variations in the morphology of the inner coma are apparent from night to night.

Images show that while many comets exhibit the approximate axial symmetry expected from radiation pressure, many more are structurally complex and lack any line of symmetry. The complex spatial structures seen in many comets are not well represented by any of the models yet described. At small heliocentric distances, comets often show continuum structures such as collimated jets and sunward fans, apparently due to 

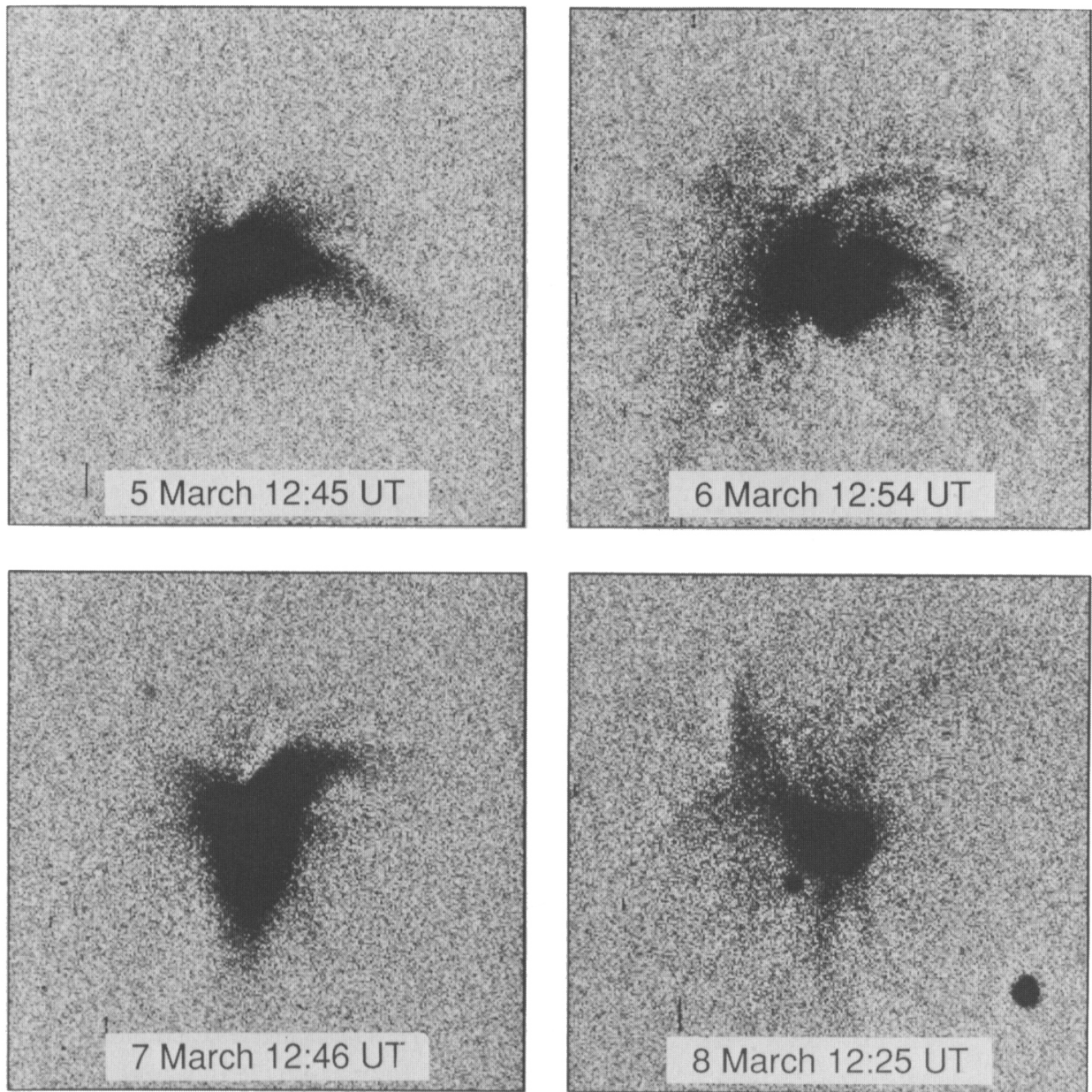

Figure 13. Same four images as shown in Figure 12, but spatially filtered to reveal fine structure. A complex system of dust jets evolves between nights. The curvature of the jets presumably reflects rotation of the nucleus, but the period(s), pole direction and rotation state of the nucleus are all controversial.

anisotropic emission from sources embedded in non-volatile surface mantles. The morphology of the continuum jets is influenced by radiation pressure, as well as by nucleus rotation. An indication of the detail contained within an active coma may be obtained by comparing Fig. 12 and Fig. 13. Spectacular examples of jets from the last 
two apparitions of comet P/Halley have been presented by Larson and Sekanina (1984) and Larson et al. (1987), but jets are a feature of many comets when at small heliocentric distances. Jets may have been detected in $\mathrm{P} / \mathrm{Halley}$ at distances as great as $R \sim 2.6 \mathrm{AU}$ (Lamy et al. 1989). Several attempts have been made, notably by Sekanina and collaborators (Sekanina 1987; Larson et al. 1987), to model the complicated jet systems seen in the inner comae of comets. This is a difficult task, since generally the nucleus rotation vector is unknown, as are the basic physical parameters of the dust in the jets (mean velocity, mean size and mean radiation-pressure acceleration). Worst of all, the ambiguity introduced by the projection of the three dimensional coma into the plane of the sky renders any interpretation non-unique.

The dust jets have a counterpart in the gas, first identified by A'Hearn et al. (1986a) (see Hoban et al. 1988). The gas jets are visible in the bands of $\mathrm{CN}$ and $\mathrm{C}_{2}$, and have a spatial distribution different from the spatial distribution of the dust jets (see Fig. 14). The gas jets may be too collimated to have a source directly rooted in the nucleus (but see Combi 1987). A plausible explanation is that the $\mathrm{CN}$ and $\mathrm{C}_{2}$ radicals are emitted by the decay of sub-micron solids, which are themselves entrained in jets. The submicron source particles remain undetected in continuum images presumably because their scattering efficiencies are low. The gas jets show clear evidence for short term variations, including evidence for rotation presumably due to the rotating nucleus. Again, exploitation of the gas jets as a route to the properties of the nucleus is a difficult matter because of the effects of projection. Figure 14 provides graphic evidence of the oversimplicity of Haser's surface brightness model.

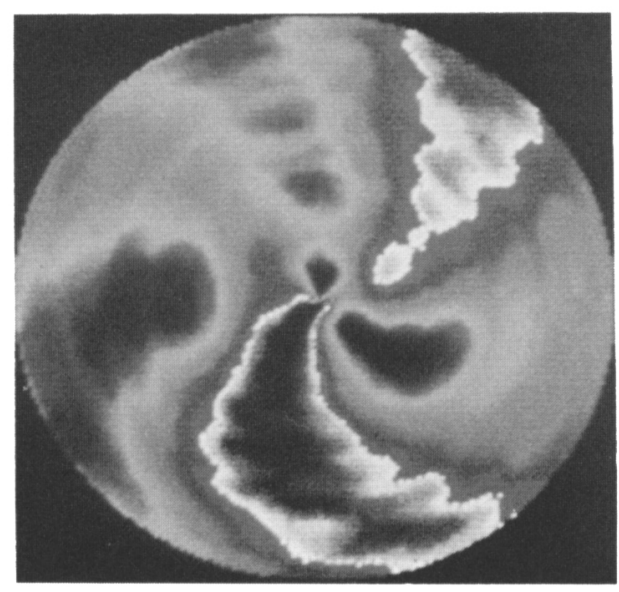

Figure 14. Image of the $\mathrm{CN}$ jets in $\mathrm{P} /$ Halley. Figure adapted from A'Hearn et al. (1986a).

Sunward fans are additional common features of the comets (Fig. 15). The fans show that mass loss from the nucleus is anisotropic, with the bulk of the mass being ejected from the hot "day side". It is possible that fans are composed of a few diffuse 
jets, and that observations at high resolution may separate these jets near their source (c.f. Keller et al. 1987). Sekanina (1987) postulates that a fan is produced in a nucleus of high obliquity when a circumpolar source is illuminated continuously by the sun. The apex angle of the cone of emission swept by the circumpolar source defines the latitude of the source, while the position angle of the cone axis gives the projection of the polar axis. The "fan model" yielded a rotation pole direction in P/Tempel 2 which was subsequently found to be compatible with the rotational lightcurve of the nucleus (Jewitt and Luu 1989). A fan model applied to $P /$ Encke may also be consistent with recent observational data (Luu and Jewitt 1990). Evidently, the fan model deserves further attention.

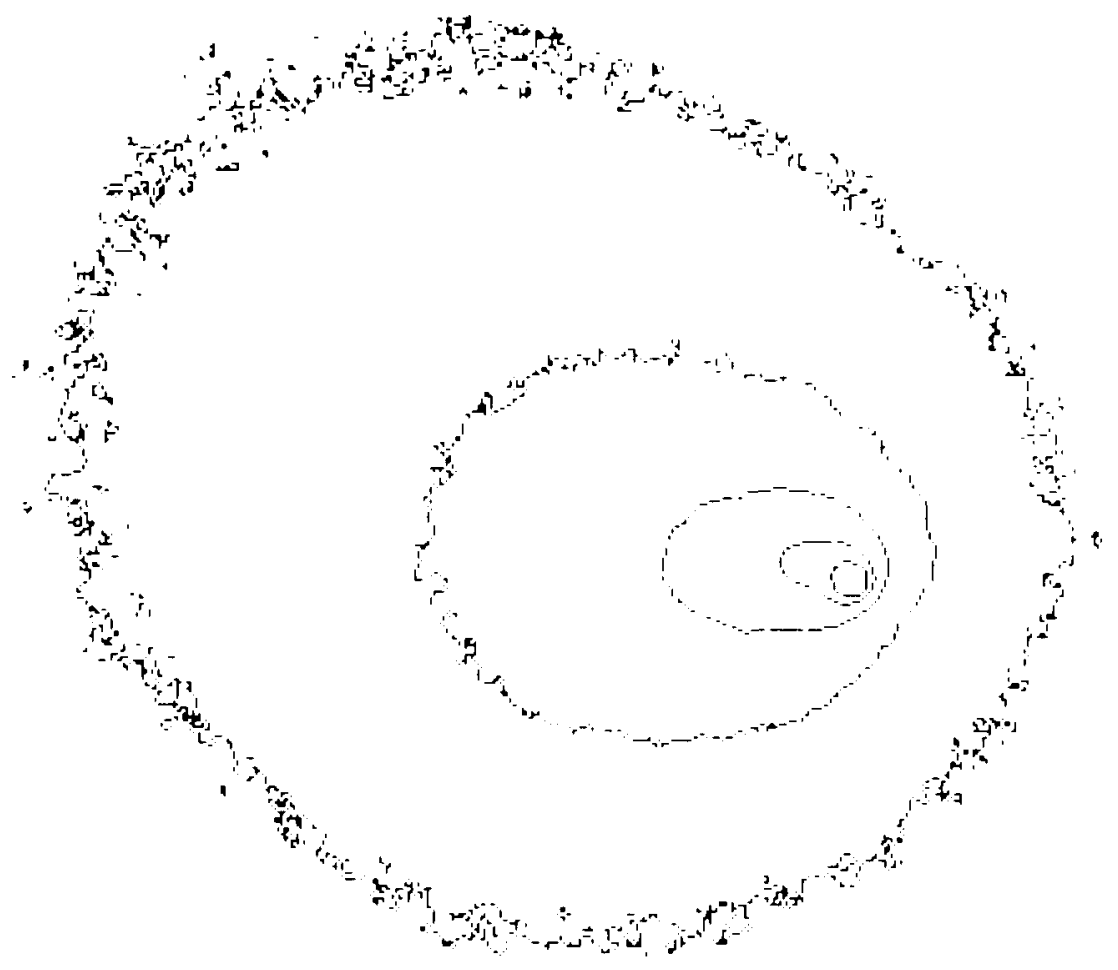

Figure 15. Example of a sunward continuum fan. This is comet IRAS/Araki/Alcock (1983d), imaged at the prime focus of the Hale 5-m telescope in an exposure of $1 \mathrm{sec}$. duration. The Sun is to the left in the figure. The field is $200 "$ across.

The structural complexity of real comae is difficult to reproduce using analytic models (e.g., Wallace and Miller 1958). Instead, recent work has focussed on the exploitation of Monte Carlo techniques. In these models, a random number generator is used to select the size, speed and direction of ejection of a dust grain from appropriate 
distributions. For example, the size may be selected from a power law distribution, while the direction of ejection may be controlled to follow any interesting distribution from isotropic to highly collimated. The trajectory of the ejected dust grain is computed including the size-dependent velocity of ejection and the acceleration due to solar radiation pressure. The position of the selected dust grain is computed at some randomly selected time of flight, and the coordinates of the grain are stored. The process is repeated for as many grains as are needed to build a high $\mathrm{S} / \mathrm{N}$ coma $\left(\sim 10^{5}-10^{6}\right.$ grains $)$. The model of the coma is completed by computing the projection of the three-dimensional coma on to the plane of the sky. The Monte Carlo models are too slow to be used in an iterative way. Their virtue is simplicity, coupled with the ease with which one can examine the effects of a change in the source function, or of the particle size distribution, for example (see Fulle 1989).

\section{Variations with Heliocentric Distance}

Interest in the heliocentric lightcurves of comets stems mainly from the desire to understand the response of the nucleus to slowly varying solar insolation. The physically interesting quantity is the variation of the mass loss rate with heliocentric distance, which can hopefully be inferred from the lightcurve by means of a model. Ideally, one would like to use measurements of the column densities of the most abundant species, water, to infer the production rates. The relative inaccessibility of the spectral features of water (from the ground), and of its immediate decay products $\mathrm{H}$ and $\mathrm{OH}$, has led to numerous attempts to use other species as indicators of the mass loss rate. Photometry of the $\mathrm{OH}$ band at $3085 \AA$ has been exploited by A'Hearn, Millis and collaborators, but is relatively neglected by other investigators. The more commonly used optical molecular species (primarily $\mathrm{CN}, \mathrm{C}_{3}, \mathrm{C}_{2}, \mathrm{NH}_{2}$ ) are trace constituents of the coma, with production rates two or more orders of magnitude beneath that of water. More often, the broad-band total magnitude is used to define the lightcurve.

The lightcurves of comets typically extend only to $R \sim 3-4 \mathrm{AU}$, since comets at larger distances are generally faint and subject to observational neglect. For physical reasons, one would like to have lightcurves that straddle $R \sim 5-6 \mathrm{AU}$, since this critical distance marks the expected outer edge of the water sublimation zone (c.f. Wyckoff et al. 1985; Meech, Jewitt and Ricker 1986; Jewitt 1990). Only a handful of comets have yet been observed systematically at these large $R$ (e.g., Figs. 3, 16 and 17), hampering any attempt to search for systematic differences between comets of different dynamical age. It is well known, however, that the lightcurves and morphologies of comets are not controlled by heliocentric distance alone -- the two comets in Fig. 16 are both at $R \sim 5$ AU and yet have very different morphologies and brightnesses. 


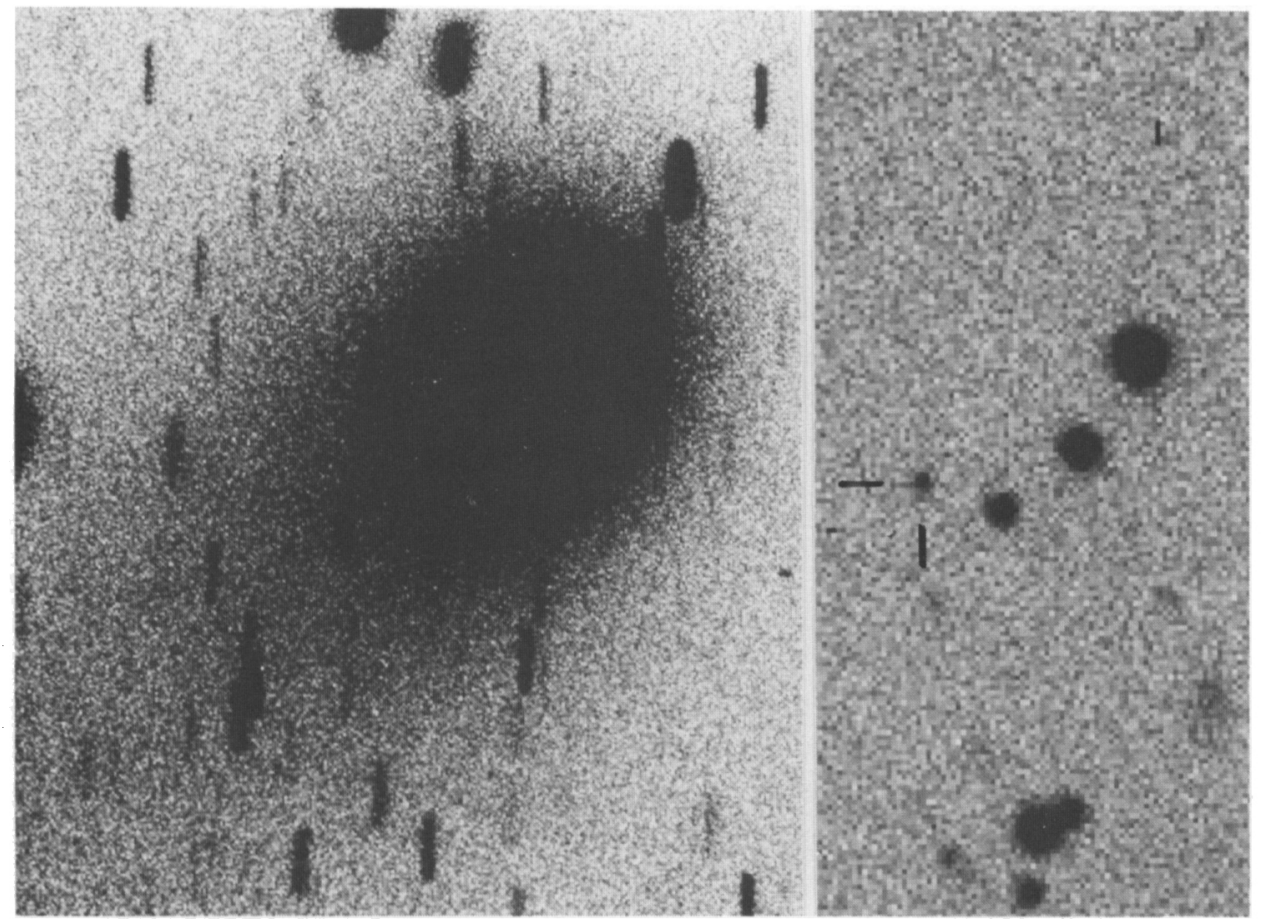

Figure 16. P/Neujmin 1 (right) at $R=5.0 \mathrm{AU}$ (UT $1986 / 3 / 6$ ) and comet Shoemaker $1984 \mathrm{f}$ (left) at $R=4.9 \mathrm{AU}$ (UT $1986 / 10 / 30$ ). Both images were taken at the Kitt Peak $2.1-\mathrm{m}$ with similar integrations and fields of view. The difference in the level of activity at the same $R$ is striking.

\subsection{MODELS}

The broad-band lightcurves of comets are frequently fitted by power laws in the heliocentric and geocentric distances

$$
H=H_{0}+2.5 n \log R+2.5 K \log \Delta
$$

where $H$ is the traditional symbol for the broadband magnitude and $H_{0}, n$ and $K$ are constants found by least squares fitting (Meisel and Morris 1982, p. 414). The case $n=2, K=2$ corresponds to the lightcurve of a point source object of constant scattering cross-section (e.g., a spherical asteroid, or comet nucleus). In photometry of an active comet with a fixed diaphragm size small compared to the size of the coma, the magnitude varies more slowly with $\Delta$ than the inverse square law predicts, because of the distributed nature of the coma. Specifically, Opik (1963) showed that $K=1$ in the presence of a strong $1 / p$ coma. Very commonly, too little information exists to permit 
a solution for either $K$ or $n$, and $K=2$ and $n=4$ are often assumed, to yield the " $H_{10}$ " magnitude

$$
H_{10}=H-10 \log R-5 \log \Delta
$$

In principle, $H_{10}$ provides a distance-corrected measure of the activity of a comet, and this quantity has been widely used for studies of the magnitude distribution and secular evolution of comets (see Meisel and Morris 1982 for examples). In practice, the assumption $K=2, n=4$ is highly questionable, and $H_{10}$ is of uncertain physical significance. Many published $H$ fail to discriminate between gas and dust, further clouding the significance of the photometry. Perhaps the strongest conclusion which can be drawn with confidence from the published investigations is that there is no compelling evidence for secular fading of comets (Meisel and Morris 1982).

Expressions of the sort in eqs. (19) and (20) have long been used to parameterize the lightcurves of comets. For instance, Whipple (1978) used measurements of $H$ versus $R$ from the literature to fit for $H_{0}, K$ and $n$ in a set of comets. He reported that the dynamically new comets had smaller $n$ than the short period, dynamically old comets, implying a physical difference between the two groups. Analyzing the same photometric data, Delsemme (1985) found no evidence for a systematic difference between the dynamically old and new comets! The difference of conclusions reflects the difficulty of extracting useful information from published photometry (most of it either visual or photographic), and the restricted range of distances covered by most lightcurves. We have already noted $(\$ 1)$ that photographic and, especially, visual magnitude estimates are difficult to interpret. Occasionally, brightness predictions based on power law extrapolations of the type in eqs. (19) and (20) fail spectacularly, as in the famous case of Comet Kohoutek 1973 XII (c.f. Öpik 1975).

Equations (19) and (20) lack physical content. What is needed is a physical model that connects the photometry with the rate of production of the dust or gas species under observation. No completely satisfactory model has yet been devised, but several useful approximations can be invoked to attempt to make physical sense of the lightcurves of comets. The Haser model is a good example of a model appropriate to photometry of the gas coma, and several gas production lightcurves have been determined using it (e.g., A'Hearn, Millis and Birch 1981; Schleicher, Millis and Birch 1987). Unfortunately, there are few gas production determinations at heliocentric distances $R>2-3 \mathrm{AU}$, and most photometry at larger distances refers to the continuum.

It is illuminating to consider a very simple dust production model, even though (or perhaps because) its defects will be immediately apparent to the reader. A related but more elaborate model has been discussed by Newburn and Spinrad (1985). In the 
present model, I use photometry from a fixed diaphragm to measure the sum of the cross sections of all dust grains within a calculable distance of the nucleus, $C_{T O T}\left[\mathrm{~m}^{2}\right]$. The total cross section is then related to the total mass of the grains, $M_{T O T}[\mathrm{~kg}]$, by a simple relation. The mass production rate is calculated by dividing $M_{T O T}$ by the time taken for a grain to travel radially from the center to the edge of the projected photometry diaphragm. To determine $M_{T O T}$, one must assume a form for the size distribution of the cometary grains. Spacecraft measurements at P/Halley suggest power-law type size distributions, such that the number of grains having radii in the range $a$ to $a+d a$ is $n(a) d a=k a^{-q} d a$, where $k$ is a constant. The magnitude of the size distribution index, $q$, varies from location to location in the Halley coma and is also a function of particle size. Values $3 \leq q \leq 4.5$ are applicable to grains in $\mathrm{P} / \mathrm{Halley}$, while the size distribution is unknown in the comae of other comets. For the present discussion, I assume that Halley-like power laws are universally applicable. Recent studies of the dust in P/Halley suggest that a significant fraction of the total mass lost from a comet is in large particles, which possess a negligible fraction of the total cross section (McDonnell et al. 1989). Therefore, the following gives a lower limit to the dust mass production rates in comets.

The total scattering cross section of the grains is

$$
C_{\text {TOT }}=\int_{a_{-}^{-}}^{a+} Q_{s} \pi a^{2} n(a) d a
$$

while the total mass is

$$
M_{T O T}=\int_{a}^{a+} \frac{4 \pi \rho a^{3} n(a) d a}{3}
$$

The mean particle size for scattering is given by

$$
\bar{a}=\frac{\int_{a-}^{a+} Q_{s} \pi a^{3} n(a) d a}{\int_{a-}^{a+} Q_{s} \pi a^{2} n(a) d a}
$$

where $a$ - and $a+$ are the minimum and maximum grain radii, respectively. Under the 
simplifying approximation, $Q_{s}=1$, eqs. (21) - (23) combine to yield the simple relation

$$
M_{T O T}=\frac{4 \rho \bar{a} C_{T O T}}{3}
$$

for the total mass in terms of the grain density, mean radius and total cross section.

The cross section $\mathrm{C}_{\mathrm{TOT}}$ may be determined from eq. (4). The phase function in eq. (4) has been measured in the range $30 \leq \alpha \leq 170$ degrees by Ney and Merrill (1976), and at small phase angles ( $0 \leq \alpha \leq 30$ degrees) by Millis et al. (1982), Kiselev and Chernova (1981), A'Hearn et al. (1984) and Meech and Jewitt (1987). The forwardscattering is strongly peaked, as would be expected from particles with $x=2 \pi a / \lambda$ $\sim 1$ (van de Hulst 1957). The back-scattering measurements are consistent with a small brightening towards zero phase, of order $0.02-0.04$ mag./deg. This is comparable to the phase coefficients measured in main-belt asteroids, and other evidence (e.g., polarization vs. phase) suggests that the coma cross section at small phases is dominated by optically large grains $(a \geq 1 \mu \mathrm{m})$. For the present, illustrative, purposes I take the mean radius as $\bar{a} \sim 1 \mu \mathrm{m}$, consistent with dynamical, thermal and other observations (c.f. Finson and Probstein 1968; Ney 1982). The adopted grain density is $\rho \sim 1000 \mathrm{~kg} \mathrm{~m}^{-3}$. Substitution into eq. (24) yields an estimate of the mass.

The time of residence of mass $M_{T O T}$ inside the photometry diaphragm is commonly called the "diaphragm crossing time". The diaphragm crossing time (in hours) is given approximately by

$$
\tau_{d} \approx 0.35 p \Delta R^{0.5}
$$

where $p$ [arcsec] is the radius of the photometry diaphragm, $R$ [AU] is the heliocentric distance and $\Delta[A U]$ is the geocentric distance. Eq. (25) was derived assuming the Bobrovnikoff-Delsemme relation (Delsemme 1982; eq. (13)) for the velocity of the coma particles. As we have previously noted, this assumption is appropriate for the micronsized grains which dominate the optical scattering from cometary dust, but it may considerably overestimate the velocity of large grains in the coma, since these may be poorly dynamically coupled to the gas.

The model mass loss rate is given by $d M / d t=M_{T O T} / \tau_{d}\left[\mathrm{~kg} \mathrm{~s}^{-1}\right]$, or 


$$
\frac{d M}{d t}=\frac{7.5 \times 10^{19} \rho \bar{a} R^{1.5} \Delta 10^{0.4\left(m_{\text {sun }} \cdot m\right)}}{p g_{\lambda} \Phi(\alpha)}
$$

It is obvious from eq. (26) that an accurate $d M / d t$ cannot be obtained, since the ground based observer does not know the quantity $\left(\rho \bar{a} / g_{\lambda} \Phi(\alpha)\right)$ to better than a factor of a few in any given comet. More importantly, the mass may be significantly underestimated if the coma contains many large particles, which may contribute little to the optical signature of the comets while containing a substantial fraction of the total mass. Evidence for very large (centimeter- to decimeter-sized) particles in comets is suggested by radar and radiocontinuum observations (e.g., Goldstein, Jurgens and Sekanina 1984; Altenhoff et al. 1986) and by Halley encounter data (McDonnell et al. 1989). With these caveats, the simple model is useful as a crude estimator of the relative $d M / d t$ among comets at a given $R$, and of variations in $d M / d t$ as a function of heliocentric distance.

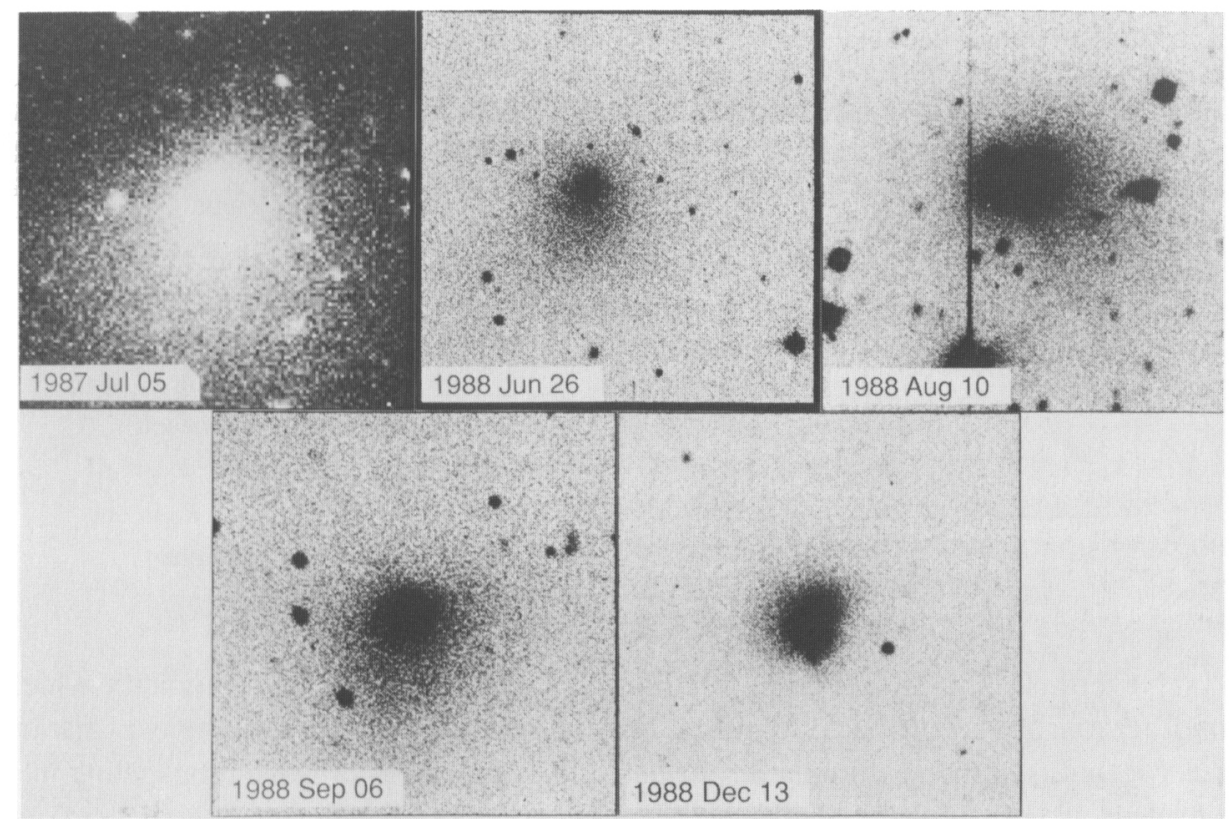

Figure 17. Activity in a comet at $R=6 \mathrm{AU}$. This is $\mathrm{P} / \mathrm{Sch}$ wassmann-Wachmann 1 imaged on five dates in 1987 and 1988. The coma, which reaches a diameter $\sim 4 \times 10^{8} \mathrm{~m}$, is a persistent feature of the comet, morphologically distinct from the more famous outbursts. Figure from Jewitt (1990). 


\subsection{OBSERVATIONS}

Many comets show strong activity at distances $R \geq 6 \mathrm{AU}$, the nominal maximum distance at which water sublimation can sustain a coma (Fig. 17). The monitoring of cometary activity has shown that the continuum and gas lightcurves of many comets are asymmetrical about perihelion. The sense of the asymmetry is sometimes reported as being positive (brighter at a given $R$ after perihelion) and sometimes negative (brighter at a given $R$ before perihelion). An apparent asymmetry (of either sign) may be caused by asymmetries in the observing geometry before and after perihelion, as we illustrate in Fig. 18 for a spherical model comet nucleus moving in the orbit of comet P/Tempel 2. Imperfect correction for geometric effects may be responsible for many of the reported cases of pre- or post-perihelion asymmetry, especially when the reported asymmetry is small (a few tenths of a mag. compared with 3 mag. in Fig. 18). There is in fact no reason to expect the apparent brightness (in gas or dust wavelengths) of a comet to ever be symmetric with respect to perihelion

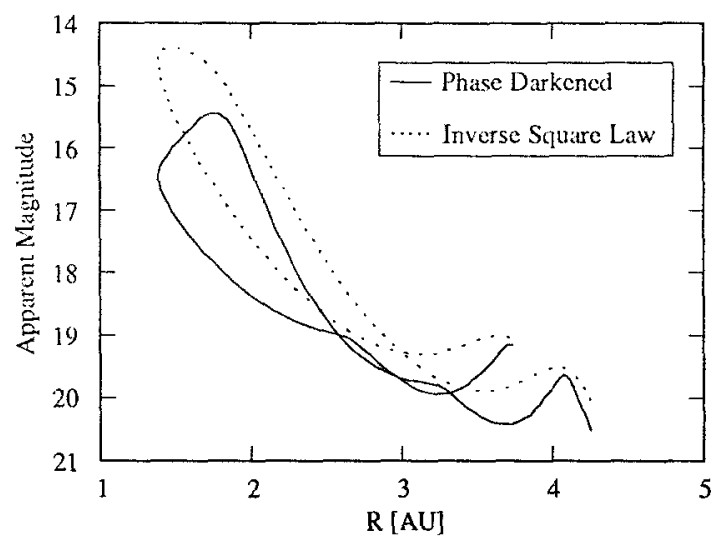

Figure 18. Lightcurve of a spherical model nucleus moving in the orbit of P/Tempel 2. The nucleus obeys the inverse square law (dashed line) and shows phase darkening with a coefficient $\beta=$ 0.04 mag. / deg (solid line). The pre-perihelion/post-perihelion asymmetry apparent at small heliocentric distance, $R$, is due entirely to viewing geometry.

Nevertheless, in a few cases, credible evidence exists for an asymmetry which cannot reasonably be attributed to geometric effects. No single compelling explanation exists for this asymmetry. It may be attributed to a slow thermal wave propagating into the nucleus and causing excess sublimation after perihelion (Fanale and Salvail 1984), or to seasonal effects caused by the non-zero obliquity and non-uniform surface activity of the nucleus. Sub-surface amorphous ice may also be warmed on the post-perihelion leg of the orbit, giving rise to the asymmetry (Smoluchowski 1985). Near-perihelion ejection of large sublimating "boulders" might also account for post-perihelion brightening (Weissman 1988). The thermal lag explanations do not account for pre-perihelion brightening, of course, but this might be explained by seasonal effects on a non-uniform nucleus. At the time of writing, the number of published explanations of the asymmetry 
is larger than the number of credible measurements of the asymmetry. I believe that it is a real effect in some comets, but much more observational and theoretical work is needed before it can be understood.

\section{Variations with Time}

There are many reasons to expect that active comets should be photometrically variable on time-scales short compared to the orbit period. The rotation of the nucleus causes a periodic heating of active areas on the surface and periodic ejection of matter into the coma. Sublimation of the surface may produce unstable topography. Collapse of the topography may yield sudden bursts of ejection, as may the exposure of volatile ices trapped in the water ice matrix. Modulation of cometary emissions by solar activity has been suggested, but no clear evidence for a connection has been demonstrated, and no convincing mechanism exists. Observational evidence in support of short-term variability comes from numerous outbursts -- sudden brightenings with amplitudes from 1 - 10 magnitudes and with durations from days to weeks. The most spectacular example of an outbursting comet is P/Schwassmann-Wachmann 1 (SW1), which is reported to flare by about 5 magnitudes to $V \sim 13$ at least several times per year (Whipple 1980; see also Jewitt 1990). Outbursts are probably common to all comets; those in SW1 are distinguished by their large size and relative frequency. Examples of short-term variability in active comets are shown in Figs. 19, 20 and 21 (see also Feldman et al. 1984; Lutz and Wagner 1986; McFadden et al. 1987).

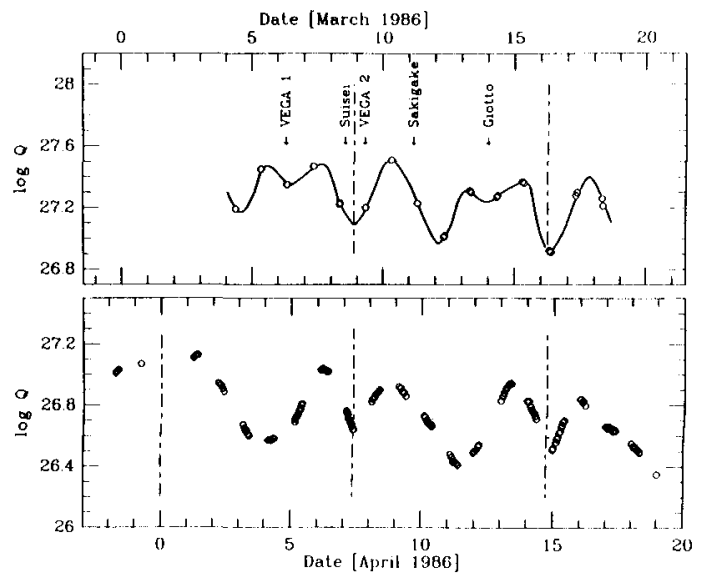

Figure 19. Temporal variability in the gas production rate from P/Halley, March and April 1986. The variations exhibit a 7.4-day period. Figure from Millis and Schleicher (1986).

The diaphragms used in photometry of comets influence the ability to detect temporal variations. The photometry from a circular diaphragm measures the sum of the scattering cross sections of the particles within the portion of the coma intercepted by the projected diaphragm. For a dust coma in which $C_{T O T}$ and $M$ are proportional (eq. 24), 
the quantity measured at instant $t_{0}$ is

$$
M\left(t_{0}\right)=\int_{t_{0}-\tau_{d}}^{t_{0}} \dot{m} d t
$$

where $\tau_{d}$ is the diaphragm crossing time (eq. (25)). The photometry diaphragm thus acts as a filter, selectively suppressing photometric variations with time scales $t \leq \tau_{d}$.

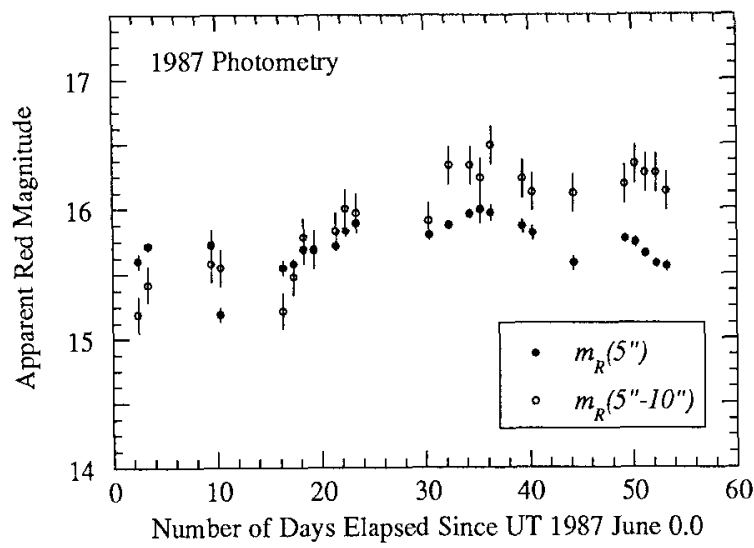

Figure 20. Temporal variability in the dust coma of P/Schwassmann-Wachmann-1, on timescales $>$ a few days. Figure from Jewitt (1990).

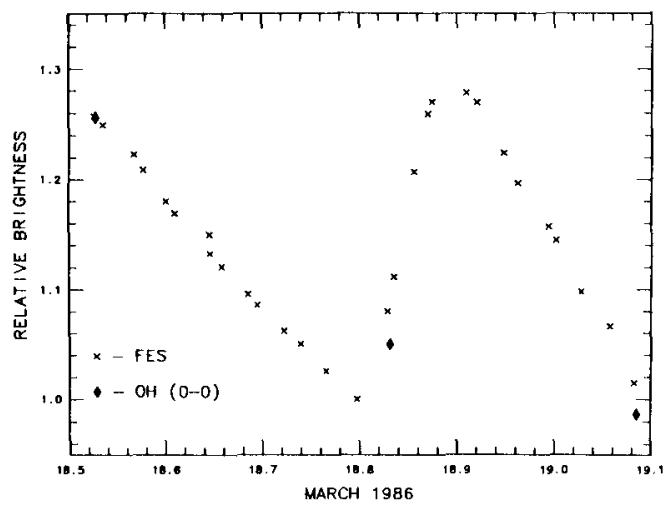

Figure 21. An outburst in $\mathrm{P} /$ Halley recorded in both continuum (labelled "FES") and gas $(\mathrm{OH})$ wavelengths. The rise time of the outburst is $<0.1$ days. Figure from McFadden et al. (1987).

Fig. 22 shows the effect of the diaphragm size on photometry of a variable, active comet. The bottom curve shows the true lightcurve of a model comet as a function of time, arbitrarily set equal to $f l u x=a+b \sin (2 \pi t / T)+\delta(t)$. The true lightcurve is approximately sinusoidal with amplitude $2 b=1 \mathrm{mag}$. and period $T$. Small ripples and outbursts (symbolized by $\delta(t)$ ) have been added to the lightcurve to simulate sporadic 
activity in the comet. The effect of photometry diaphragms of increasing size is shown by the upper curves. For the case in which the diaphragm crossing time is one tenth the period of variation, $\tau_{d} / T=0.1$, the lightcurve has already lost much of the fine structure seen in the bottom curve. With $\tau_{d} / T=0.5$, even the major outburst near $t / T=0.5$ has been lost, and at $\tau_{d} / T=1.0$ there is basically no evidence for any variation. Note also the systematic phase shift in the lightcurves as larger diaphragms are used (Fig. 22).

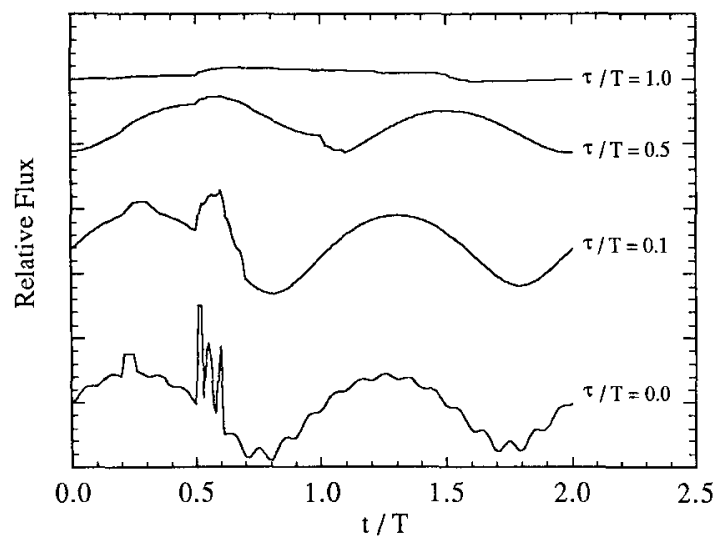

Figure 22. Effect of the size of the photometry diaphragm on the measured temporal variations in an active comet. The bottom curve shows a model light-curve, including a cyclic component (period $T$ ) plus several "outbursts". The upper curves show the lightcurves resulting from the use of successively larger photometry diaphragms. $\tau / T$ is the diaphragm crossing time in units of the period of the fundamental variation.

Based on eq. (27) (and Fig. 22) we may expect to observe periodic modulation of the coma by the rotating nucleus only if $\tau_{d} / T<<1$, where $T$ is the rotation period. Periodic variations most likely due to nucleus rotation have indeed been observed in P/Halley (Fig. 19), and are suspected in P/Schwassmann-Wachmann 1 (Fig. 20). However, cyclic coma variations are absent in other comae in which they have been sought, probably because $\tau_{d} / T \ll 1$ is not satisfied in many comets. For instance, the average nucleus rotation period appears to be near $T \sim 12 \mathrm{hrs}(\$ 3)$. A 10"-radius photometry diaphragm, used to observe a comet at $R=\Delta=2 \mathrm{AU}$, gives $\tau_{d} \sim 10 \mathrm{hrs}$, which does not satisfy $\tau_{d} / T \ll 1$. Only in special cases in which a very small projected diaphragm size is possible (e.g., in good seeing) or in which the period of the nucleus is very long, can the effects of rotation be detected in photometry of the coma. In the case of P/Halley the inferred period ( $T \sim 7$ days) is much larger than the average diaphragm crossing time $\tau_{d} \sim 0.5$ days (a 39" radius diaphragm was used by Millis and Schleicher (1986); see also Neckel and Munch 1987), so that $\tau_{d} / T \ll 1$ is easily satisfied. 
Evidence for ultra-rapid photometric variations (on time-scales $\ll \tau_{d}$ ) has been reported by Isserstedt and Schlosser (1975). Their reported 4-minute periodicity has not been explained -- the similarity with the 5-minute solar global oscillation period as discussed by these authors is almost certainly devoid of physical significance. High time resolution measurements of $\mathrm{P} /$ Halley (Rettig et al. 1987) show no significant variations on 1 - minute to 10 - minute time-scales, exactly as expected from our discussion of eq. (27).

\section{Variations with Wavelength}

An excellent review of the general optical properties of dust in comets determined up to the mid-1980's may be found in Lamy (1986). More recent findings are summarized here. The normalized gradient of the reflectivity, $S^{\prime}\left(\lambda_{1}, \lambda_{2}\right)$, provides a useful measure of the continuum color

$$
S^{\prime}\left(\lambda_{1}, \lambda_{2}\right)=\frac{1}{\bar{S}} \frac{\partial S}{\partial \lambda}
$$

where $\bar{S}$ is the mean reflectivity within wavelength range $\lambda_{1} \leq \lambda \leq \lambda_{2}$. The reflectivity gradient, $S^{\prime}$, is conveniently expressed in per-cent per $1000 \AA$. The reflectivity gradients vary from comet to comet, but they also vary with wavelength (Jewitt and Meech 1986; Lamy et al. 1989) and with position on the coma. Figure 23 summarizes the wavelength dependence of $S^{\prime}$ in the optical and near-IR wavelength regions. $S^{\prime}$ is positive in the visual (i.e. comet dust is red). The trend toward neutral and blue colors at long wavelengths is consistent with a geometrical effect in scattering from small particles ( $a \sim 1 \mu \mathrm{m}$; Jewitt and Meech 1986) but may also be an effect of composition in scattering from macroscopic grains (Lamy 1989). Hartmann and Cruikshank (1984) and Hanner and Newburn (1989) reported that the continuum color varies with heliocentric distance, but, using an independent and homogeneous data set, Jewitt and Meech $(1986,1988$ a) found no evidence for a color-distance, or a color-phase angle trend. The dispersion in $S^{\prime}$ at a given wavelength in Fig. 23 appears to indicate real color differences among the comets, rather than an artifact of the observing geometry. It is not known whether the color differences are caused by comet to comet variations in the size distributions of grains, or by compositional differences, or both (Jewitt and Meech 1988a). Unfortunately, frequency histograms of the J-H, H-K colors of comets show no evidence for distinct color groups, of the kind seen in asteroid color data. Thus, no basis presently exists for a classification of the comets founded on their continuum colors, either in the optical or in the near-IR. 


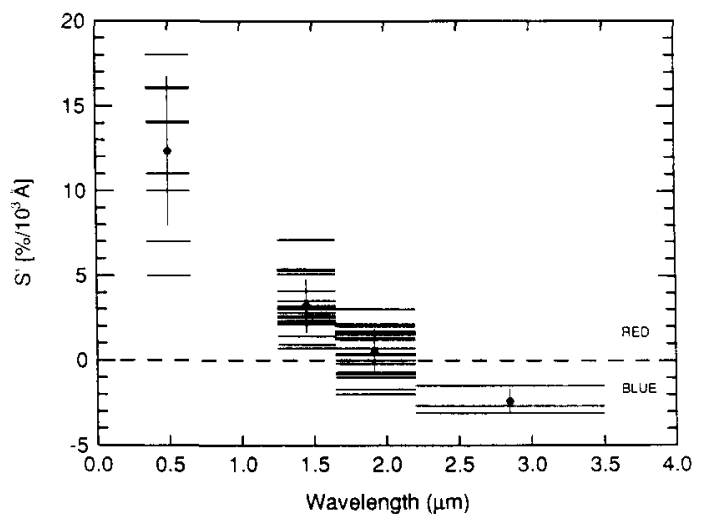

Figure 23. Reflectivity gradient of cometary dust comae plotted as a function of wavelength. Neutral scattering is indicated by $S^{\prime}=0$. The reflectivity gradient is a decreasing function of increasing wavelength in the range $0.4 \leq \lambda \leq 3.5 \mu \mathrm{m}$. Figure from Jewitt and Meech (1986).

Optical evidence for spatial variations in the continuum color has been presented by Jewitt and Meech (1986) and by Hoban et al. (1989). These variations may result from spatial variations in the mean particle size and/or composition, which, in turn, may be a natural consequence of radiation-pressure sorting. Modification of grains while in flight (e.g., by sublimation) and even inhomogeneous dust sources in the nucleus may play a role in the observed color variations. Recently, it has been found that the cometary dust may have a different color from that of the nucleus. For example, the nucleus of $\mathrm{P} / \mathrm{Halley}$ was slightly bluer than the near-nucleus coma (Thomas and Keller 1989), while the nucleus of $\mathrm{P} / \mathrm{Tempel} 2$ was markedly redder than the surrounding neutral coma (A'Hearn et al. 1989; Jewitt and Luu 1989). These puzzling observations might be explained by multiple scattering in the nucleus and/or by Mie single-particle scattering in the coma grains.

\section{Summary of Outstanding Problems}

Despite the central importance of photometry to the understanding of comets, there is, in fact, relatively little reliable photometric work in the literature to provide the badly needed context for modern studies. I end this review by listing some basic cometary problems which await solution by photometry:

(1). What are the physical properties of cometary nuclei? Are there differences in the size, shape, color or rotation period distributions of nuclei compared to these quantities in the asteroids? Can the above properties be related to the different collisional environments in which the comets and asteroids exist? Ground-based photometry represents the only available route to the study of nuclei as a group (\$3). 
(2). Which, if any, of the nucleus properties are primordial? The obvious test of the effect of solar insolation is to compare the nuclei of dynamically new and old comets. To date, only the nuclei of dynamically old comets have been measured. Photometry of the nuclei of dynamically new comets represents a potentially rewarding, but extremely difficult, challenge. Dynamically new comets are discovered by virtue of their activity, effectively precluding direct study of their nuclei.

(3). What factors control the rates of growth of mantles on cometary nuclei? Can the mantled fraction be used as an indicator of "exposure age"? The optical properties of the nuclei are those of their mantles. Photometry at a variety of wavelengths might be used to diagnose the mantle compositions in different comets. Do new comets possess surface mantles due to cosmic ray processing, or are these mantles self-destroyed by exothermic reactions at large $R$ ?

(4). Can mantle coverage reach $100 \%$, and if so, do nuclei choke themselves to death, leaving inert, asteroid-like remnant bodies? There is room for a cometary contribution to the known population of Near-Earth Asteroids, but no specific evidence that dead comets actually exist among the NEAs. A photometric study might confirm the link by revealing physical similarities (size, shape, color and albedo) between heavily mantled nuclei and NEAs.

(5). What are the physical properties of comets at large heliocentric distances? A majority of physical observations of comets are taken at small heliocentric distances, where the morphology of the comet is dominated by sublimation of water ice on the nucleus. At larger distances, the water sublimation rate is small, leaving other volatiles and perhaps other physical processes to drive the coma. Recent evidence suggests that comae can be detected in some comets well beyond the orbit of Saturn. What are the physical properties of these distant comae, and what produces them?

(6). What are the grain coma expansion velocities? The BobrovnikoffDelsemme relation (eq. 14) is derived from observations of expanding structures in the inner comae of comets. The physical nature of the structures (are they gas or dust?) is unclear. Therefore, the grain expansion velocities are actually quite uncertain. Basic quantities which depend on the expansion velocity, for instance the dust production rates (eq. 26), are likewise rendered uncertain.

(7). Can water IGHs be detected? Current best estimates give the water IGH scale lengths as $100-1000 \mathrm{~km}$ at $R=1 \mathrm{AU}(\$ 4.4)$, subtending angles $\theta \sim 1 "$. Careful analysis of images from a good site might reveal the water IGH.

(8). Are fading grains ubiquitous among comets? The steep surface brightness 
profiles of some comae (Baum and Kreidl 1986; Jewitt and Meech 1987a; Jewitt and Luu 1989), and the detection of $\mathrm{C}_{2}$ and $\mathrm{CN}$ jets (A'Hearn et al. 1986b), are both consistent with the idea that cometary grains may disintegrate with increasing time of flight from the nucleus (see Boehnhardt and Fechtig 1987). Do all comets possess fading grains? What fraction of cometary volatiles is released from coma grains rather than from the nucleus directly?

(9). How do the properties of the nucleus influence the properties of the coma? What are the effects of nucleus obliquity, rotation, asphericity, surface mantling and heat conduction on the appearance of the coma? Initial observations suggest that the influence varies among comets from strong (e.g., in P/Halley, where the coma photometry varies strongly in response to nucleus rotation) to weak (e.g., in P/Tempel 2 , where the coma photometry is independent of the rotation of the underlying nucleus). Existing data (e.g., Figs. 13 and 14) merely hint at the complexity of the interaction between nucleus and coma. If the interactions can be deciphered, it should be possible to work backwards from the observed morphology of the coma to the properties of the nucleus.

\section{Acknowledgments}

I am grateful for financial support provided by past and present grants from the National Science Foundation and the National Aeronautics and Space Administration. I thank M. A'Hearn, J. Luu, R. Remillard and an anonymous individual for their reviews.

\section{REFERENCES}

A'Hearn, M.F. (1982). 'Spectrophotometry of Comets at Optical Wavelengths,' in Comets, edited by L.L. Wilkening, University of Arizona Press, Tucson, pp. 433460.

A'Hearn, M.F. (1983). 'Photometry of Comets,' in Solar System Photometry Handbook, edited by R. Genet, Willmann-Bell, Richmond, Virginia, p. 3-1.

A'Hearn, M.F., Millis, R.L., and Birch, P.V. (1981). 'Comet Bradfield 1979X: The Gassiest Comet?', Astron. J., 86, 1559-1566.

A'Hearn, M.F., Schleicher, D.G., Feldman, P.D., Millis, R.L., and Thompson, D.T. (1984). 'Comet Bowell 1980b,' Astron. J., 89, 579-591.

A'Hearn, M.F., Hoban, S., Birch, P.V., Bowers, C., Martin, R., and Klinglesmith, D.A. (1986a). 'CN Jets in Comet P/Halley,' Nature, 324, 649-651.

A'Hearn, M.F., Hoban, S., Birch, P.V., Bowers, C., Martin, R., and Klinglesmith, D.A. (1986b). 'Gaseous Jets in Comet P/Halley,' in 20th ESLAB Symposium, ESA SP-250, Heidelberg, pp. 483-486.

A'Hearn, M.F., Campins, H., Schleicher, D.G., and Millis, R.L. (1989). 'The Nucleus of P/Tempel 2,' Astrophys. J., 347, 1155-1166.

Altenhoff, W.J., Huchtmeier, W.K., Schmidt, J., Schraml, J.B., Stumpff, P., and Thum, C. (1986). 'Radio Continuum Observations of Comet Halley,' Astron. Astrophys., 164, 227-230. 
Baum, W.A., and Kreidl, T.J. (1986). 'Volatiles in Cometary Grains,' in Asteroids, Comets, Meteors II, edited by C.-I. Lagerkvist, B.A. Lindblad, H. Lundstedt, and H. Rickman, Uppsala University Press, Uppsala, pp. 397-402.

Baum, W.A., Thomsen, B., and Kreidl, T.J. (1981). 'Subtleties in the Flat-Fielding of CCD Images,' in Solid State Imagers for Astronomy, Proc. Int. Soc. Opt. Eng., 290, 24-27.

Belton, M.J.S. (1990). 'Rationalization of Halley's Periods: Evidence for an Inhomogeneous Nucleus?', Icarus, in press.

Boehnhardt, H., and Fechtig, H. (1987). 'Electrostatic Charging and Fragmentation of Dust Near P/Giacobini-Zinner and P/Halley,' Astron. Astrophys., 187, 824-828.

Bowell, E., Hapke, B., Domingue, D., Lumme, K., Peltoniemi, J., and Harris, A.W. (1989). 'Application of Photometric Models to Asteroids,' in Asteroids II, edited by R.P. Binzel, T. Gehrels, and M.S. Matthews, University of Arizona Press, Tucson, pp. 524-556.

Burns, J.A., Lamy, P.L., and Soter, S. (1979). 'Radiation Forces in the Solar System,' Icarus, 40, 1-48.

Campins, H., A'Hearn, M.F., and McFadden, L. (1987). 'The Bare Nucleus of Comet P/Neujmin 1,' Astrophys. J., 316, 847-857.

Cochran, A. (1985). 'A Re-Evaluation of the Haser Model Scale Lengths for Comets,' Astron. J., 90, 2609-2614.

Combi, M.R. (1987). 'Sources of Cometary Radicals and Their Jets: Gases or Grains?', Icarus, 71, 178-191.

Combi, M.R., and Smyth, W.H. (1988). 'Monte Carlo Particle-Trajectory Models for Neutral Cometary Gases. I. Models and Equations,' Astrophys. J., 327, 10261043.

Delsemme, A.H. (1976). 'The Neutral Coma of Comets: A Review,' in The Study of Comets, edited by B. Donn, M. Mumma, W. Jackson, M. A'Hearn, and R. Harrington, NASA SP-393, U.S. Government Printing Office, Washington, D.C., pp. 711-737.

Delsemme, A.H. (1982). 'Chemical Composition of Cometary Nuclei,' in Comets, edited by L.L. Wilkening, University of Arizona Press, Tucson, pp. 85-130.

Delsemme, A.H. (1985). 'The Sublimation Temperature of the Cometary Nucleus,' in Ices in the Solar System, edited by J. Klinger, D. Benest, A. Dollfus, and R. Smoluchowski, NATO Advanced Science Institutes Series, C 156, D. Reidel Pub. Co., Dordrecht, p. 367.

Delsemme, A.H., and Miller, D.C. (1971). 'Physico-Chemical Phenomena in CometsIII. The Continuum of Comet Burnham (1960 II), Planet. Space Sci., 19, 12291257.

Dermott, S.F., Harris, A.W., and Murray, C.D. (1984). 'Asteroid Rotation Rates,' Icarus, 57, 14-34.

Dollfus, A., and Suchail, J.-L. (1987). 'Polarimetry of Grains in the Coma of Comet P/Halley. I. Observations,' Astron. Astrophys., 187, 669-688.

Eddington, A.S. (1910). 'The Envelopes of Comet Morehouse (1908c),' M. N. R. A. S., 70, 442-458.

Fanale, F.P., and Salvail, J.R. (1984). 'An Idealized Short-Period Comet Model: Surface Insolation, $\mathrm{H}_{2} \mathrm{O}$ Flux, Dust Flux, and Mantle Evolution,' Icarus, 60, 476-511. 
Fay, T.D., and Wisniewski, W. (1978). 'The Lightcurve of the Nucleus of Comet d'Arrest,' Icarus, 34, 1-9.

Feldman, P.D., A'Hearn, M.F., and Millis, R.L. (1984). 'Temporal and Spatial Behavior of the Ultraviolet Emissions of Comet IRAS-Araki-Alcock 1983d,' Astrophys. J., 282, 799-802.

Festou, M.C. (1981). 'The Density Distribution of Neutral Compounds in Cometary Atmospheres. I. Models and Equations,' Astron. Astrophys., 95, 69-79.

Festou, M.C., and Feldman, P.D. (1981). 'The Forbidden Oxygen Lines in Comets,' Astron. Astrophys., 103, 154-159.

Finson, M.L., and Probstein, R.F. (1968). 'A Theory of Dust Comets. I. Model and Equations,' Astrophys. J., 154, 327-352.

Fort, B. (1985). 'New Trends in CCD Photometry of Galaxies,' in New Aspects of Galaxy Photometry, edited by H. Araki, Springer-Verlag, Berlin, pp. 3-12.

Fulle, M. (1989). 'Evaluation of Cometary Dust Parameters From Numerical Simulations: Comparison With Analytical Approach and Role of Anisotropic Emissions,' Astron. Astrophys., 217, 283-297.

Gammelgaard, P., and Thomsen, B. (1988). ' $\mathrm{C}_{2}, \mathrm{H}_{2} \mathrm{O}^{+}$, and Dust Distribution in Comet P/Halley Near Opposition,' Astron. Astrophys., 197, 320-326.

Goldstein, R.M., Jurgens, R.F., and Sekanina, Z. (1984). 'A Radar Study of Comet 1983d,' Astron. J., 89, 1745-1754.

Gombosi, T.I. (1986). 'A Heuristic Model of the Comet Halley Dust Size Distribution,' in 20th ESLAB Symposium, ESA SP-250, Heidelberg, pp. 167-171.

Gunn, J., and Westphal, J.A. (1981). 'Care, Feeding, and Use of Charge-Coupled Device (CCD) Imagers at Palomar Observatory,' in Solid State Imagers for Astronomy, Proc. Int. Soc. Opt. Eng., 290, 16-23.

Hanner, M.S. (1981). 'On the Detectability of Icy Grains in the Comae of Comets,' Icarus, 47, 342-350.

Hanner, M.S., and Newburn, R. (1989). 'Infrared Photometry of Comet Wilson (1986I) at Two Epochs,' Astron. J., 97, 254-261.

Hartmann, W.K., and Cruikshank, D. (1984). 'Comet Color Changes With Solar Distance,' Icarus, 57, 55-62.

Haser, L. (1957). 'Distribution D'Intensitie Dans la Tete d'une Comete,' Bull. Acad. Roy. Sci. Liege, 43, 740.

Haser, L. (1966). 'Calcul de Distribution D'Intensitie Relative Dans une Tete Cometaire,' Mem. Soc. Roy. Sci. Liege, XII, 233-242.

Hellmich, R., and Keller, H.U. (1981). 'On the Visibility of Nuclei of Dusty Comets,' Icarus, 47, 325-332.

Hoban, S. Samarasinha, N.H., A'Hearn, M.F., and Klinglesmith, D.A. (1988). 'An Investigation Into Periodicities of CN Jets in Comet P/Halley,' Astron. Astrophys., $195,331-337$.

Hoban, S., A'Hearn, M.F., Birch, P.V., and Martin, R. (1989). 'Spatial Structure in the Color of the Dust Coma of Comet P/Halley,' Icarus, 79, 145-158.

Isserstedt, J., and Schlosser, W. (1975). 'Intensity Fluctuations in the Head of Comet 1973f,' Astron. Astrophys., 41, 9-13.

Jewitt, D.C. (1990). 'The Persistent Coma of Comet P/Schwassmann-Wachmann 1,' Astrophys. J., in press. 
Jewitt, D.C., and Danielson, G.E. (1984). 'Charge Coupled Device Photometry of Comet P/Halley,' Icarus, 60, 435-444.

Jewitt, D.C., and Luu, J.X. (1989). 'A CCD Portrait of Comet P/Tempel 2,' Astron. J., 97, 1766-1790.

Jewitt, D.C., and Meech, K.J. (1985). 'Rotation of the Nucleus of P/Arend-Rigaux,' Icarus, 64, 329-335.

Jewitt, D.C., and Meech, K.J. (1986). 'Cometary Grain Scattering Versus Wavelength, or, "What Color Is Comet Dust?",' Astrophys. J., 310, 937-952.

Jewitt, D.C., and Meech, K.J. (1987a). 'Surface Brightness Profiles of 10 Comets,' Astrophys. J., 317, 992-1001.

Jewitt, D.C., and Meech, K.J. (1987b). 'CCD Photometry of Comet P/Encke,' Astron. J., 93, 1542-1548.

Jewitt, D.C., and Meech, K.J. (1988a). 'The Absence of a Color-Distance Trend in Comets,' Astron. J., 96, 1723-1730.

Jewitt, D.C., and Meech, K.J. (1988b). 'Optical Properties of Cometary Nuclei and a Preliminary Comparison With Asteroids,' Astrophys. J., 328, 974-986.

Keller, H.U., et al. (1987). 'Comet Halley's Nucleus and Its Activity,' Astron. Astrophys., 187, 807-823.

Kiselev, N.N., and Chernova, G.P. (1981). 'Phase Functions of Polarization and Brightness and the Nature of Cometary Atmosphere Particles,' Icarus, 48, 473481.

Lamy, P.L. (1986). 'Cometary Dust: Observational Evidences and Properties,' in Asteroids, Comets, Meteors II, edited by C.-I. Lagerkvist, B.A. Lindblad, H. Lundstedt, and H. Rickman, Uppsala University Press, Uppsala, pp. 373-388.

Lamy, P.L. (1989). Presentation at "Comets in the Post-Halley Era," Bamberg.

Lamy, P.L., and Perrin, J.-M. (1988). 'Optical Properties of Organic Grains: Implications for Interplanetary and Cometary Dust,' Icarus, 76, 100-109.

Lamy, P.L., Pederson, H., and Vio, R. (1987). 'The Dust Tail of Comet P/Halley in April 1986,' Astron. Astrophys., 187, 661-664.

Lamy, P.L., Malburet, P., Llebaria, A., and Koutchmy, S. (1989). 'Comet P/Halley at a Heliocentric Preperihelion Distance of 2.6 AU: Jet Activity and Properties of the Coma,' Astron. Astrophys., 222, 316-322.

Larson, S.M. (1986). 'A Review of Some Digital Image Processing in Cometary Research,' in Asteroids, Comets, Meteors II, edited by C.-I. Lagerkvist, B.A. Lindblad, H. Lundstedt, and H. Rickman, Uppsala University Press, Uppsala, pp. $449-459$.

Larson, S.M., and A'Hearn, M.F. (1984). 'Comet Bowell (1980b): Measurement of the Optical Thickness of the Coma and Particle Albedo From Stellar Occultation,' Icarus, 58, 446-450.

Larson, S.M., and Sekanina, Z. (1984). 'Coma Morphology and Dust Emission Pattern of P/Halley. I. High Resolution Images Taken at Mount Wilson in 1910,' Astron. J., 89, 571-578.

Larson, S.M., Sekanina, Z., Levy, D., Tapia, S., and Senay, M. (1987). 'Comet P/Halley Near-Nucleus Phenomena in 1986,' Astron. Astrophys., 187, 639-644.

Luppino, G.A. (1989). 'A Virtual Phase CCD Camera for MDM Observatory,' P. A. S. P., 101, 931-939. 
Lutz, B.L., and Wagner, R.M. (1986). 'Temporal Variability of Comet IRAS-ARAKIALCOCK 1983d: The May 10 Event,' Astrophys. J., 308, 993-1000.

Luu, J.X. (1988). 'The Scale Lengths of Molecules in P/Halley,' unpublished manuscript. Luu, J.X., and Jewitt, D.C. (1990). 'The Nucleus of Comet P/Encke,' Icarus, in press.

Massonne, L., Fertig, J., Grün, E., and Schwehm, G. (1986). 'A Cometary Dust Environment Model for the Generation of Synthetic Images,' in Asteroids. Comets. Meteors II, edited by C.-I. Lagerkvist, B.A. Lindblad, H. Lundstedt, and H. Rickman, Uppsala University Press, Uppsala, pp. 407-410.

McDonnell, J.A.M., Pankiewicz, G.S., Birchley, P.N.W., Green, S.F., and Perry, C.H. (1989). 'The Comet Nucleus: Ice and Dust Morphological Balances in a Production Surface of Comet P/Halley,' submitted to Proc. XXth L. P. S. C.

McFadden, L.A., A'Hearn, M.F., Feldman, P.D., Roettger, E.E., Edsall, D.M., and Butterworth, P.S. (1987). 'Activity of Comet P/Halley 23-25 March, 1986: IUE Observations,' Astron. Astrophys., 187, 333-338.

Meech, K.J., and Jewitt, D.C. (1987). 'Observations of Comet P/Halley at Minimum Phase Angle,' Astron. Astrophys., 187, 585-593.

Meech, K.J., Jewitt, D.C., and Ricker, G. (1986). 'Early Photometry of Comet P/Halley: Development of the Coma,' Icarus, 66, 561-574.

Meisel, D.D., and Morris, C.S. (1982). 'Comet Head Photometry: Past, Present and Future,' in Comets, edited by L.L. Wilkening, University of Arizona Press, Tucson, pp. 413-432.

Mendis, D.A., and Ip, W.-H. (1976). 'The Neutral Atmospheres of Comets,' Astrophys. Space Sci., 39, 335-385.

Meredith, N.P., Wallis, M.K., and Rees, D. (1989). 'The Anomalous Behavior of $\mathrm{C}_{2}$ in P/Borrelly 1987p,' M. N. R. A. S., 240, 647-655.

Millis, R.L., and Schleicher, D.G. (1986). 'Rotational Period of Comet Halley,' Nature, $324,646-649$.

Millis, R.L., A'Hearn, M.F., and Thompson, D.T. (1982). 'Narrowband Photometry of Comet P/Stephan-Oterma and the Backscattering Properties of Cometary Grains,' Astron. J., 87, 1310-1317.

Millis, R.L., A'Hearn, M.F., and Campins, H. (1988). 'An Investigation of the Nucleus and Coma of Comet P/Arend-Rigaux,' Astrophys. J., 324, 1194-1209.

Mukai, T., Mukai, S., Fechtig, H., Grun, E., and Giese, R.H. (1985). 'Dirty Ice Grains in Comets,' Adv. Space Res., 5, 339-342.

Neckel, T., and Munch, G. (1987). 'Photometry of Comet Halley at Near Post-Perihelion Phases,' Astron. Astrophys., 187, 581-584.

Newburn, R., and Spinrad, H. (1985). 'Spectrophotometry of Seventeen Comets II. The Continuum,' Astron. J., 90, 2591-2608.

Newburn, R., and Spinrad, H. (1989). 'Spectrophotometry of 25 Comets,' Astron. J., 97, 552-569.

Ney, E.P. (1982). 'Visibility of Comet Nuclei,' Science, 215, 397-398.

Ney, E.P., and Merrill, K. (1976). 'Comet West and the Phase Function of Cometary Dust,' Science, 194, 1051-1053.

Notni, P., and Tiersch, H. (1987). 'Charging of Dust Particles in Comets and in Interplanetary Space,' Astron. Astrophys., 187, 796-800.

O'Dell, C.R. (1971). 'Nature of Particulate Matter in Comets as Determined From Infrared Observations,' Astrophys. J., 166, 675-681. 
O'Dell, C.R., Robinson, R.R., Krishna Swamy, K.S., McCarthy, P.J., and Spinrad, H. (1988). $\mathrm{C}_{2}$ in Comet Halley: Evidence for Its Being Third Generation and Resolution of the Vibrational Population Discrepancy,' Astrophys. J., 334, 476488.

Öpik, E.J. (1963). 'Photometry, Dimensions and Ablation Rate of Comets,' Irish Astr. J., $6,93-112$.

Öpik, E.J. (1975). 'On Predicting the Brightness of Comets,' in Comet Kohoutek, edited by G.A. Gary, NASA SP-355, Washington, D.C., pp. 205-207.

Padgham, C.A., and Saunders, J.E. (1975). The Perception of Light and Colour, G. Bell and Sons, London.

Rees, D., Meredith, N.P., and Wallis, M.K. (1986). 'Optical Observations of the Neutral and Ion Coma of Comet Halley,' in 20th ESLAB Symposium, ESA SP-250, Heidelberg, pp. 493-498.

Rettig, T.W., Kern, J.R., Ruchti, R., Baumbaugh, B., Baumbaugh, A.E., Knickerbocker, A.E., and Dawe, J. (1987). 'Observations of the Coma of Comet P/Halley and the Outburst of 1986 March 24-25 (UT),' Astron. Astrophys., 187, 249-255.

Roemer, E. (1966a). 'Cometary Nuclei-Introductory Report,' Mem. Soc. Roy. Sci. Liege, XII, 15-22.

Roemer, E. (1966b). 'The Dimensions of Cometary Nuclei,' Mem. Soc. Roy. Sci. Liege, XII, 23-28.

Roemer, E. (1976). 'Luminosity and Astrometry of Comets: A Review,' in The Study of Comets, edited by B. Donn, M. Mumma, W. Jackson, M. A'Hearn, and R. Harrington, NASA SP-393, U.S. Government Printing Office, Washington, D.C., pp. $380-409$.

Russell, H.N. (1916). 'On the Albedo of the Planets and Their Satellites,' Astrophys. J., 43, 173-195.

Salo, H. (1988). 'Monte Carlo Modelling of the Net Effects of Coma Scattering and Thermal Reradiation on the Energy Input to the Cometary Nucleus,' Icarus, 76, 253-269.

Schleicher, D.G., Millis, R.L., and Birch, P.V. (1987). 'Photometric Observations of Comet P/Giacobini-Zinner,' Astron. Astrophys., 187, 531-538.

Sekanina, Z. (1976). 'A Continuing Controversy: Has the Cometary Nucleus Been Resolved?', in The Study of Comets, edited by B. Donn, M. Mumma, W. Jackson, M. A'Hearn, and R. Harrington, NASA SP-393, U.S. Government Printing Office, Washington, D.C., pp. 537-587.

Sekanina, Z. (1987). 'Anisotropic Emission From Comets: Fans Versus Jets I. Concept and Modeling,' in Symposium on the Diversity and Similarity of Comets, ESA SP-278, Brussels, Belgium.

Sekanina, Z., and Farrell, J.A. (1982). 'Two Dust Populations of Particle Fragments in the Striated Tail of Comet Mrkos 1957 V,' Astron. J., 87, 1836-1853.

Simpson, J.A., Rabinowitz, D., Tuzzolino, A.J., Ksanfomality, L.V., and Sagdeev, R.Z. (1987). 'The Dust Coma of Comet P/Halley: Measurements on the Vega-1 and Vega-2 Spacecraft,' Astron. Astrophys., 187, 742-752. 
Smoluchowski, R. (1985). 'Amorphous and Porous Ices in Cometary Nuclei,' in Ices in the Solar System, edited by J. Klinger, D. Benest, A. Dollfus, and R. Smoluchowski, NATO Advanced Science Institutes Series, C 156, D. Reidel Pub. Co., Dordrecht, pp. 397-406.

Thomas, N., and Keller, H.U. (1989). "The Colour of Comet P/Halley's Nucleus and Dust,' Astron. Astrophys., 213, 487-494.

van de Hulst, H.C. (1957). Light Scattering by Small Particles, Wiley, New York.

Vaisberg, O.L., Smirnov, V., Omelchenko, A., Gorn, L., and Iovlev, M. (1987). 'Spatial and Mass Distribution of Low-Mass Dust Particles $\left(\mathrm{m}<10^{-10} \mathrm{~kg}\right)$ in Comet P/Halley's Coma,' Astron. Astrophys., 187, 753-760.

Wallace, L.V., and Miller, F.D. (1958). 'Isophote Configurations for Model Comets,' Astron. J., 63, 213-219.

Wallis, M.K., Rabilizirov, R., and Wickramasinghe, N.C. (1987). 'Evaporating Grains in P/Halley's Coma,' Astron. Astrophys., 187, 801-806.

Weissman, P.R. (1988). 'Why Was Halley's Comet So Bright?', B. A. A. S., 20, 825.

Weissman, P.R., and Kieffer, H.H. (1981). 'Thermal Modelling of Cometary Nuclei,' Icarus, 47, 302-311.

West, R.N., and Jorgenson, H.E. (1989). 'Post-Perihelion Observations of Comet P/Halley at $\mathrm{R}=8.5 \mathrm{AU}$,' Astron. Astrophys., 218, 307-316.

West, R.N., Pedersen, H., Monderen, P., Vio, R., and Grosbol, P. (1986). 'PostPerihelion Imaging of Comet Halley at ESO,' Nature, 321, 363-365.

Whipple, F.L. (1978). 'Cometary Brightness Variation and Nucleus Structure,' Moon and Planets, $18,343-359$.

Whipple, F.L. (1980). 'Rotation and Outbursts of Comet P/SW1,' Astron. J., 85, 305313.

Whipple, F.L. (1982). 'The Rotation of Comets,' in Comets, edited by L.L. Wilkening, University of Arizona Press, Tucson, pp. 227-250.

Whipple, F.L. (1989). 'Comets in the Space Age,' Astrophys. J., 341, 1-15.

Whipple, F.L., and Sekanina, Z. (1979). 'Comet Encke: Precession of the Spin Axis, Non-Gravitational Motion, and Sublimation,' Astron. J., 84, 1894-1909.

Wilhelm, K. (1986). 'Rotation and Precession of Comet Halley,' Nature, 327, 27-30.

Wyckoff, S., Wagner, R.M., Wehinger, P.A., Schleicher, D.G., and Festou, M.C. (1985). 'Onset of Sublimation in Comet P/Halley (1982i),' Nature, 316, 241-242. 